Linköping Studies in Science and Technology.

Dissertations. No. 1730

\title{
Topology optimization considering stress, fatigue and load uncertainties
}

\author{
Erik Holmberg
}

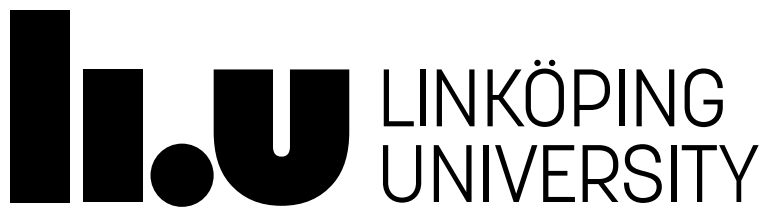

Division of Solid Mechanics

Department of Management and Engineering

Linköping University, SE-581 83, Linköping, Sweden

Linköping, November 2015 
Cover:

Optimized topology of an L-shaped beam, obtained by minimizing the mass subjected to stress constraints. The design domain and boundary conditions are given in Figure 7.

Printed by:

LiU-Tryck, Linköping, Sweden, 2015

ISBN 978-91-7685-883-7

ISSN 0345-7524

Distributed by:

Linköping University

Department of Management and Engineering

SE-581 83, Linköping, Sweden

\section{(C) 2015 Erik Holmberg}

This document was prepared with $\mathrm{AT}_{\mathrm{E} X}$, November 25, 2015

No part of this publication may be reproduced, stored in a retrieval system, or be transmitted, in any form or by any means, electronic, mechanical, photocopying, recording, or otherwise, without prior permission of the author. 


\section{Preface}

The work presented in this dissertation has been carried out at Saab AB and at the Division of Solid Mechanics, Linköping University. The research has been supported by NFFP (nationella flygtekniska forskningsprogrammet) Grant No. 201301221, which is funded by the Swedish Armed Forces, the Swedish Defence Materiel Administration and the Swedish Governmental Agency for Innovation Systems.

I am very grateful for the guiding from my supervisors, Anders Klarbring and Bo Torstenfelt, and I have very much appreciated all interesting and fruitful discussions, with them and Carl-Johan Thore, about structural optimization and finite element analysis. I would also like to thank colleagues at Saab AB and Linköping University for encouragement and interesting work-related, as well as off-topic, discussions. Finally, I would also like to express my gratitude for the daily support from my lovely wife Elina and our wonderful son Sixten.

\section{Erik Holmberg}

Linköping, November 2015 



\section{Abstract}

This dissertation concerns structural topology optimization in conceptual design stages. The objective of the project has been to identify and solve problems that prevent structural topology optimization from being used in a broader sense in the avionic industry; therefore the main focus has been on stress and fatigue constraints and robustness with respect to load uncertainties.

The thesis consists of two parts. The first part gives an introduction to topology optimization, describes the new contributions developed within this project and motivates why these are important. The second part includes five papers.

The first paper deals with stress constraints and a clustered approach is presented where stress constraints are applied to stress clusters, instead of being defined for each point of the structure. Different approaches for how to create and update the clusters, such that sufficiently accurate representations of the local stresses are obtained at a reasonable computational cost, are developed and evaluated.

High-cycle fatigue constraints are developed in the second paper, where loads described by a variable-amplitude load spectrum and material data from fatigue tests are used to determine a limit stress, for which below fatigue failure is not expected. A clustered approach is then used to constrain the tensile principal stresses below this limit.

The third paper introduces load uncertainties and stiffness optimization considering the worst possible loading is then formulated as a semi-definite programming problem, which is solved very efficiently. The load is due to acceleration of point masses attached to the structure and the mass of the structure itself, and the uncertainty concerns the direction of the acceleration. The fourth paper introduces an extension to the formulated semi-definite programming problem such that both fixed and uncertain loads can be optimized for simultaneously.

Game theory is used in the fifth paper to formulate a general framework, allowing essentially any differentiable objective and constraint functions, for topology optimization under load uncertainty. Two players, one controlling the structure and one the loads, are in conflict such that a solution to the game, a Nash equilibrium, is a design optimized for the worst possible load. 



\section{Populärvetenkaplig sammanfattning}

Låg vikt och effektivare produktutveckling är intressant i många industriella tillämpningar, speciellt inom flygindustrin. Genom att använda strukturoptimering tidigt i produktutvecklingsprocessen kan en betydande andel vikt sparas jämfört med traditionell, manuell, design. Dessutom, om strukturen redan i tidigt skede är optimerad med avseende på de strukturella krav som ställs senare i processen så minskar antalet designändringar som krävs, vilket gör processen effektivare.

Topologioptimering är en form av strukturoptimering som lämpar sig att använda tidigt i produktutvecklingsprocessen. I topologioptimering utgår man inte ifrån en befintlig design utan man definierar ett designområde, som strukturen ska hålla sig inom, och utifrån de laster som strukturen ska bära och hur den monteras hittar optimeringen en struktur som uppfyller bivillkoren och där målfunktionen är minimerad. Resultatet är en mer eller mindre grov modell, som används som grund för att skapa den geometriska modell som traditionellt är det första steget i produktutvecklingsprocessen.

För att den optimerade strukturen ska vara användbar och inte kräva stora ändringar i senare designskeden måste de krav som ställs på strukturen vara beaktade redan i topologioptimeringen.

Denna avhandling presenterar metoder för hur bivillkor på spänning och utmattning kan användas i topologioptimering och även metoder för hur man får en design som är robust mot osäkra laster. Spänningsbivillkor innebär att strukturen skapas på ett sådant sätt att massan minimeras utan att ett statiskt brott uppstår på grund av de pålagda lasterna. Utmattningsbivillkor innebär att strukturen även ska hålla för upprepade belastningar, där alla laster strukturen utsätts för under den beräknade livslängden beaktas.

Med robusthet avses här att optimeringen hittar den värsta lasten som kan uppstå och optimerar strukturen för den lasten, strukturens prestanda är därför bättre eller lika bra för alla andra laster. Strukturen är därmed robust, eftersom ingen last kan få förödande konsekvenser, vilket kan vara fallet om bara en, eller ett fåtal, lastfall beaktas. 



\section{List of Papers}

The following five papers have been included in this thesis:

I. E. Holmberg, B. Torstenfelt, A. Klarbring (2013). Stress constrained topology optimization. Structural and Multidisciplinary Optimization, 48(1):33-47.

II. E. Holmberg, B. Torstenfelt, A. Klarbring (2014). Fatigue constrained topology optimization. Structural and Multidisciplinary Optimization, 50(2):207219.

III. E. Holmberg, C-J. Thore, A. Klarbring (2015). Worst-case topology optimization of self-weight loaded structures using semi-definite programming. Structural and Multidisciplinary Optimization, DOI 10.1007/s00158-015-1285-1.

IV. C-J. Thore, E. Holmberg, A. Klarbring (2015). Large-scale robust topology optimization under load-uncertainty. In proceedings: 11th World Congress on Structural and Multidisciplinary Optimization. Sydney, Australia.

V. E. Holmberg, C-J. Thore, A. Klarbring (2015). Game theory approach to robust topology optimization with uncertain loading. Submitted.

Own contribution

I have been the main contributor in writing all papers but paper IV, for which my contribution in writing is mainly proof reading and commenting. The theory in all papers is developed in collaboration with the co-authors. The main part of the implementation in the first two papers is made in collaboration with Bo Torstenfelt, and the implementation and software development in the last three papers is made by me. In all papers I have created the numerical models and performed the optimizations. 



\section{Contents}

Preface $\quad$ iii

Abstract $\quad$ v

Populärvetenkaplig sammanfattning vii

List of Papers ix

Contents $\quad$ xi

Part I - Theory and background 1

1 Introduction 3

2 Structural optimization 5

2.1 Different optimization areas: size, shape and topology . . . . . . . . 5

2.1.1 Short historical background to topology optimization . . . . 6

2.2 Solving the optimization problem . . . . . . . . . . . . . . 7

2.3 Sensitivity analysis . . . . . . . . . . . . . . . 8

3 Discretization of the continuum problem 11

3.1 Design variables . . . . . . . . . . . . . . . . . . . 11

3.2 Numerical instabilities . . . . . . . . . . . . . . . . . . . . 12

3.3 Filtering techniques . . . . . . . . . . . . . . . . . . . . 14

3.4 Obtaining a black-and-white design . . . . . . . . . . . . . . . 15

3.5 Stress singularity and penalization of stresses . . . . . . . . . . . . 16

3.6 Mass penalization . . . . . . . . . . . . . . . . . . 18

4 Stress and fatigue constraints $\quad 19$

4.1 On the importance of using constraints adapted to the application . 19

4.2 Stress constraints . . . . . . . . . . . . . . . . 20

4.2.1 Global and clustered stress measures . . . . . . . . . . . 22

4.3 High-cycle fatigue . . . . . . . . . . . . . . . . . . . . . . . . . 24

4.3.1 Load spectrum and material data . . . . . . . . . . . 25

4.3.2 Fatigue analysis . . . . . . . . . . . . . . . . 27 
4.3.3 Fatigue constraints . . . . . . . . . . . . . . 28

5 Optimization under load uncertainty 33

5.1 Semi-definite programming approach . . . . . . . . . . . . . . 33

5.1.1 Comparison with eigenvalue formulation . . . . . . . . 35

5.2 Game theory approach . . . . . . . . . . . . . . . . 37

6 Software 43

6.1 Developed FEM and optimization program . . . . . . . . . . . . 43

$\begin{array}{lll}7 & \text { Industrial application } & \mathbf{4 7}\end{array}$

7.1 Topology optimization of a landing gear part . . . . . . . . . . . . . 47

8 Summary and conclusions $\quad 53$

9 Review of included papers $\quad 55$

$\begin{array}{lc}\text { Bibliography } & 63\end{array}$

Part II - Included papers $\quad 65$

Paper I: Stress constrained topology optimization . . . . . . . . . . . . 69

Paper II: Fatigue constrained topology optimization . . . . . . . . . . . 87

Paper III: Worst-case topology optimization of self-weight loaded struc-

tures using semi-definite programming . . . . . . . . . . . 103

Paper IV: Large-scale robust topology optimization under load-uncertainty 119

Paper V: Game theory approach to robust topology optimization with

uncertain loading . . . . . . . . . . . . . . . . . . 127 


\section{Part I}

\section{Theory and background}





\section{Introduction}

Light weight designs are desirable in many industrial applications, in particular within the avionic and automotive industries. Decreasing the structural mass of aircrafts, cars and trucks has several immediate results, such as improved performance and reduced fuel consumption which in turn gives reduced emissions and an increased range. A lighter design also gives the possibility to increase pay-load.

The work presented in this thesis strives to generate lighter designs in a conceptual design phase. This is achieved by the use of topology optimization, for which methods have been developed such that the result is a mature optimized design that will require as little manual work as possible in later design phases. As this work concerns the mathematical, rather than the organizational, framework of optimization, a simplified design chain is sufficient to describe how the optimizations presented in this thesis fit into the product development process; the conceptual design gives a first basic idea of the design and all remaining design phases are here denoted detailed design.

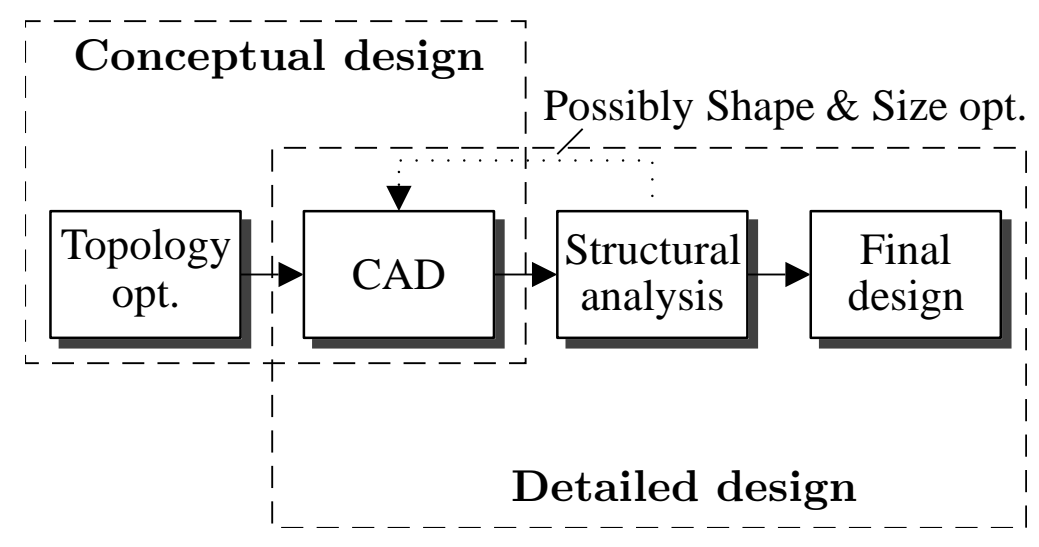

Figure 1: Simplified product development process involving topology optimization methods that are well adapted to the industrial problem.

By introducing structural optimization at an early stage of the product development process, a lighter design can be achieved without necessarily increasing the 


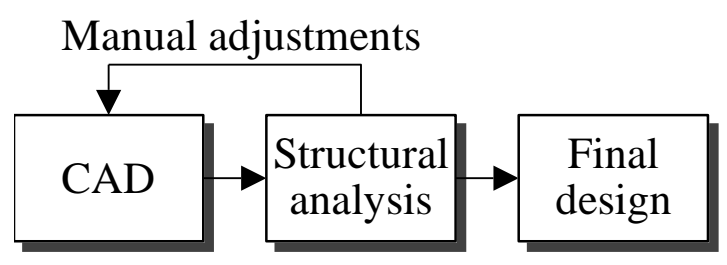

Figure 2: Simplified manual product development process, without any optimization.

amount of development work required. The basic idea is that if structural optimization is well incorporated into the methods used for product development, the process can be made much faster than conventional product development, as the number of manual iterations between the "design office" and "structural analysis office" are significantly reduced, or perhaps even eliminated. A simplified flowchart of a product development process is shown in Figure 1, and can be compared to the simplified flowchart for manual design shown in Figure 2; at least one step is added, but the need for time consuming trial-and-error in the form of structural analysis followed by manual re-design is reduced. This means that a reduction of working time, and thus of development costs, are other positive effects of using structural optimization.

The work presented in this thesis contributes with methods for using constraints involving allowable stresses and fatigue life in topology optimization. It also confronts one of the main problems of optimization in general and of topology optimization in particular, namely that an optimized design is traditionally not robust and may not be able to carry any other loads than those for which it was optimized. This has been done by introducing uncertainties in the applied load. These uncertainties can be actual uncertainties involving loads that are expected to occur, or fictitious uncertainties that are introduced to make the design more robust in general, so that it stands a better chance to withstand unexpected loads due to handling or redesign in a later design stage. 


\section{Structural optimization}

Structural optimization is a general term involving several techniques used to optimize the design of a load carrying structure. The design variables $\boldsymbol{x}$ influence the design of the structure under consideration and these are updated in an automated way such that the objective function $g_{0}(\boldsymbol{x})$ is minimized and the constraints are satisfied.

A general structural optimization problem reads

$$
(\mathbb{P})\left\{\begin{array}{l}
\min _{\boldsymbol{x} \in \mathbb{R}^{m}} g_{0}(\boldsymbol{x}) \\
\text { s.t. }\left\{\begin{array}{l}
g_{i}(\boldsymbol{x}) \leq \bar{g}_{i}, i=1, \ldots, c, \\
\underline{x}_{e} \leq x_{e} \leq \bar{x}_{e}, e=1, \ldots, m,
\end{array}\right.
\end{array}\right.
$$

where "s.t." reads "subject to"; the $c$ constraints state that the functions $g_{i}(\boldsymbol{x})$ need to return a value not greater than the upper limits $\bar{g}_{i}$ and the $m$ design variables have the lower and upper bounds $\underline{x}_{e}$ and $\bar{x}_{e}$, respectively.

In this thesis, the functions $g_{j}(\boldsymbol{x}), j=0, \ldots, c$ are calculated using the finite element method (FEM) [18] for linearly elastic structures. A nested formulation is used, meaning that the nodal displacement vector $\boldsymbol{u} \in \mathbb{R}^{n}$, where $n$ is the number of degrees of freedom of the model, is found as the solution of the state equation

$$
\boldsymbol{K}(\boldsymbol{x}) \boldsymbol{u}=\boldsymbol{f}(\boldsymbol{x}, \boldsymbol{r}),
$$

where the stiffness matrix $\boldsymbol{K}(\boldsymbol{x}) \in \mathbb{R}^{n \times n}$ is influenced by the design variables and $\boldsymbol{f}(\boldsymbol{x}, \boldsymbol{r}) \in \mathbb{R}^{n}$ is the force vector, which may be influenced by the design variables and which may also be controlled by some external vector $\boldsymbol{r}$. This means that the stiffness matrix needs to be invertible, so that the displacements become implicit functions of $\boldsymbol{x}$, i.e. $\boldsymbol{u}=\boldsymbol{u}(\boldsymbol{x})=\boldsymbol{K}(\boldsymbol{x})^{-1} \boldsymbol{f}(\boldsymbol{x}, \boldsymbol{r})$.

\subsection{Different optimization areas: size, shape and topology}

Structural optimization is usually divided into three main areas: size, shape and topology optimization. In size and shape optimization, an existing design is parameterized by, usually, a moderate number of design variables, and finding an 
optimized design often means that the end of the design chain is reached. That is, the optimized design constitutes the design as it will be manufactured. In size optimization, the design variables can control parameters such as the cross-sectional area of a beam or the thickness of a plate and a fixed FE-mesh is used. In shape optimization, the variables influence the shape of the discretized structure by modifying the shape of the elements, e.g. using pre-defined shapes parameterized using spline functions.

In contrast to size and shape optimization, topology optimization does not start from a known design and usually does not generate a structural design that is ready for manufacturing; instead, it generates a very first conceptual design that needs to be interpreted into a geometric model before its performance can be evaluated with greater confidence. A design domain that defines the geometric restrictions on the design is defined, and the entire design domain is discretized by a finite element mesh. A design variable is related to each element in the design domain and determines if the element will represent structural material or a hole. The connectivity of the structure, while connecting the applied loads to the given supports, is thus changed such that the objective function is minimized subjected to the specified constraints. This means that the number of design variables in a topology optimization problem is typically very large. Compared to size and shape optimization, topology optimization allows more freedom, as it does not rely on an existing and typically non-optimal design, and therefore topology optimization allows for the greatest gain in performance.

The focus of this thesis is on topology optimization.

\subsubsection{Short historical background to topology optimization}

The first steps towards what today is called topology optimization were made in the mid 1960s, when a number of papers on optimization of truss structures were published, e.g. by Dorn et al. [22]. The first practical implementation of optimization in the form of point-wise material or voids on a fixed finite element mesh, in order to obtain an optimized shape, was performed by Bendsøe and Kikuchi in 1988 [5]. This was based on earlier work by e.g. Kohn and Strang in 1986 [56], [32], but was also inspired by papers concerning optimization of the thickness distribution of elastic plates, such as that by Cheng and Olhoff from 1981 [15].

The concept of topology optimization that is common today, i.e. penalization of stiffness for intermediate design variable values in order to achieve a design with only solid material and voids, was introduced by Bendsøe in 1989 [4] and it was later named SIMP, Solid Isotropic Material with Penalization, by Rozvany [48]. An extensive historical discussion of the SIMP method is given by Rozvany in [45] and [46]. The word topology optimization originates from the Greek word topos which means landscape or place [52].

From the very beginning, topology optimization has been synonymous with finding 
the stiffest topology, given a limited amount of material. Such a problem is formulated as minimizing the compliance $C(\boldsymbol{x}, \boldsymbol{r})=1 / 2 \boldsymbol{f}(\boldsymbol{x}, \boldsymbol{r})^{\top} \boldsymbol{u}$; the lower the compliance, the higher the stiffness for the loads $\boldsymbol{f}(\boldsymbol{x}, \boldsymbol{r})$. This traditional minimum compliance formulation has gained its popularity much because the compliance is a convex function when $\boldsymbol{K}(\boldsymbol{x})$ depends linearly on $\boldsymbol{x}$ and because $C(\boldsymbol{x}, \boldsymbol{r})$ is a so-called self-adjoint function, as will be described in Chapter 2.3, which makes it computationally efficient.

\subsection{Solving the optimization problem}

There are a number of different methods for solving $(\mathbb{P})$; the choice depends mainly on the nature of the functions involved. Problems involving noisy functions, or functions that are non-differentiable due to e.g. re-meshing of the FE-model, are often solved using Response Surface Methodology (RSM) [38]. In RSM, metamodels, or response surfaces, are built by running FE-analyses with different settings on the design variables and evaluating the function values $g_{j}(\boldsymbol{x}), j=0, \ldots, c$. A response surface is built by a polynomial based on the function values for the different $\boldsymbol{x}$ such that it approximates the response of the real model as well as possible. Optimization may then be performed on the response surfaces rather than on the full model. Obviously, if the number of variables is large, the cost of creating a response surface will be overwhelming. Moreover, an error is introduced when a response surface is created, so this method should only be used when no other method is available.

This work focuses on differentiable functions which are solved using first order, gradient-based methods. In these methods gradients of the functions $g_{j}(\boldsymbol{x})$ with respect to the design variables $\boldsymbol{x}$ are calculated, upon which design variable changes are determined. How these gradients, or sensitivities, are calculated is the topic of the following section.

Two different optimization solvers have been used in this work: the Method of Moving Asymptotes (MMA) developed by Svanberg [58]; and the interior-pointsolver IPOPT [67]. Structural optimization problems are generally non-convex, which is why optimization solvers typically create convex approximations at the current design $\boldsymbol{x}$. These approximations are only reasonably accurate for small design updates, which is why the size of the step is restricted and the optimization problems are solved in an iterative manner. In this work, relatively fine convergence criteria and conservative settings of the solvers have been used, so that no numerical problems with the solvers are expected.

As a note, there are non-gradient based topology optimization methods, but motivated by the critical review by Sigmund [54], these methods are not discussed in this work. 


\subsection{Sensitivity analysis}

The structural optimization problem $(\mathbb{P})$ is often solved using a first-order method, where an initial design is updated in an iterative manner, based on gradients $\partial g_{j}(\boldsymbol{x}) / \partial x_{e}, j=0, \ldots, c, e=1, \ldots, m$. The computation of these gradients is called sensitivity analysis and is performed using either approximate, numerical methods or, exact, analytical methods. In the numerical analysis, the sensitivity of $g_{j}(\boldsymbol{x})$ with respect to one variable is approximated by giving it a small perturbation and performing a new FE-analysis with this value, the response is then compared to that obtained for the nominal value. This means that numerical gradients can be used if the functions are not available analytically, typically if the FE-solver is used as a "black box". However, at least one additional linear system (1) needs to be solved for each variable, making this method expensive for problems involving a large number of design variables.

Analytical sensitivity analysis means that the functions $g_{j}(\boldsymbol{x}), j=0, \ldots, c$ are differentiable and available in the formulation, such that they may be differentiated analytically and then implemented into the optimization and FE software.

In structural optimization, the objective function and the constraint functions are often dependent on the displacements. Due to the nested formulation, the displacements are implicitly functions of $\boldsymbol{x}$, meaning that the functions may be written as $g_{j}(\boldsymbol{x})=\hat{g}_{j}(\boldsymbol{x}, \boldsymbol{u}(\boldsymbol{x}))$. The sensitivity with respect to design variable $x_{e}$ follows from the product rule and the chain rule, and reads

$$
\frac{\partial g_{j}(\boldsymbol{x})}{\partial x_{e}}=\frac{\partial \hat{g}_{j}(\boldsymbol{x}, \boldsymbol{u}(\boldsymbol{x}))}{\partial x_{e}}+\frac{\partial \hat{g}_{j}(\boldsymbol{x}, \boldsymbol{u}(\boldsymbol{x}))}{\partial \boldsymbol{u}(\boldsymbol{x})} \frac{\partial \boldsymbol{u}(\boldsymbol{x})}{\partial x_{e}} .
$$

The sensitivity of the displacements is calculated by differentiating the state equation (1):

$$
\frac{\partial \boldsymbol{K}(\boldsymbol{x})}{\partial x_{e}} \boldsymbol{u}(\boldsymbol{x})+\boldsymbol{K}(\boldsymbol{x}) \frac{\partial \boldsymbol{u}(\boldsymbol{x})}{\partial x_{e}}=\frac{\partial \boldsymbol{f}(\boldsymbol{x}, \boldsymbol{r})}{\partial x_{e}},
$$

which after rearrangement of terms reads

$$
\frac{\partial \boldsymbol{u}(\boldsymbol{x})}{\partial x_{e}}=\boldsymbol{K}(\boldsymbol{x})^{-1}\left[\frac{\partial \boldsymbol{f}(\boldsymbol{x}, \boldsymbol{r})}{\partial x_{e}}-\frac{\partial \boldsymbol{K}(\boldsymbol{x})}{\partial x_{e}} \boldsymbol{u}(\boldsymbol{x})\right] .
$$

Inserting (3) into (2) yields

$$
\begin{aligned}
\frac{\partial g_{j}(\boldsymbol{x})}{\partial x_{e}}=\frac{\partial \hat{g}_{j}(\boldsymbol{x}, \boldsymbol{u}(\boldsymbol{x}))}{\partial x_{e}} & \\
& +\frac{\partial \hat{g}_{j}(\boldsymbol{x}, \boldsymbol{u}(\boldsymbol{x}))}{\partial \boldsymbol{u}(\boldsymbol{x})} \boldsymbol{K}(\boldsymbol{x})^{-1}\left[\frac{\partial \boldsymbol{f}(\boldsymbol{x}, \boldsymbol{r})}{\partial x_{e}}-\frac{\partial \boldsymbol{K}(\boldsymbol{x})}{\partial x_{e}} \boldsymbol{u}(\boldsymbol{x})\right] .
\end{aligned}
$$

This formulation (4) is known as the direct method. Here we find that the linear system (3) needs to be solved $m$ times - once for each design variable. Therefore, 
obtaining the sensitivities in (4) will be too expensive if the number of design variables is large.

For problems involving a large number of design variables the adjoint method is preferable. In the adjoint method, another set of linear systems is created from (4) by introducing the vector $\boldsymbol{\lambda}_{j}$ as

$$
\boldsymbol{\lambda}_{j}^{\top}=\frac{\partial \hat{g}_{j}(\boldsymbol{x}, \boldsymbol{u}(\boldsymbol{x}))}{\partial \boldsymbol{u}(\boldsymbol{x})} \boldsymbol{K}(\boldsymbol{x})^{-1} .
$$

The linear system in (5) needs to be solved $c+1$ times $^{1}$ and $\boldsymbol{\lambda}_{j}^{\top}$ is then inserted into (4), which gives the adjoint sensitivity formulation:

$$
\frac{\partial g_{j}(\boldsymbol{x})}{\partial x_{e}}=\frac{\partial \hat{g}_{j}(\boldsymbol{x}, \boldsymbol{u}(\boldsymbol{x}))}{\partial x_{e}}+\boldsymbol{\lambda}_{j}^{\top}\left[\frac{\partial \boldsymbol{f}(\boldsymbol{x}, \boldsymbol{r})}{\partial x_{e}}-\frac{\partial \boldsymbol{K}(\boldsymbol{x})}{\partial x_{e}} \boldsymbol{u}(\boldsymbol{x})\right] .
$$

From (4) and (6) it is clear that the direct method is to be preferred when the number of constraints is greater than the number of design variables, and that the adjoint method is preferable for the opposite situation.

The popularity of the traditional compliance formulation hinges partly on its self-adjoint property: with $\hat{g}_{j}(\boldsymbol{x}, \boldsymbol{u}(\boldsymbol{x}))=1 / 2 \boldsymbol{f}(\boldsymbol{x}, \boldsymbol{r})^{\top} \boldsymbol{u}$, the gradient with respect to $\boldsymbol{u}(\boldsymbol{x})$ times the inverse of the stiffness matrix in (4) and (5) becomes $1 / 2 \boldsymbol{f}(\boldsymbol{x}, \boldsymbol{r})^{\top} \boldsymbol{K}(\boldsymbol{x})^{-1}=1 / 2 \boldsymbol{u}^{\top}$, where the equality follows from (1). Thus, no additional linear system needs to be calculated to obtain the gradients.

For topology optimization problems, analytical gradients and the adjoint method are used almost exclusively - this is the case also in this thesis.

\footnotetext{
${ }^{1}$ Usually, less than $c+1$ are required as not all $g_{j}(\boldsymbol{x}), j=0, \ldots, c$ are functions of $\boldsymbol{u}$.
} 



\section{Discretization of the continuum problem}

In structural topology optimization, the finite element method is used to discretize the continuum problem and to solve the static equilibrium problem (1). The finite element mesh is also used to define the design variables. The continuum problem is defined on the design domain $\Omega$, which in topology optimization often has a box shape, as in Figure 3, within which the structure should be contained.

\subsection{Design variables}

One design variable, $x_{e}$, is used for each finite element that discretizes the design domain $\Omega$. The design variables are often initially presented as discrete variables, where the value is either 1 , implying that the element represents material, or 0 meaning that the element represents a hole. Thus, the design variables determine the connectivity of the structure within the given design domain.

However, for the large number of design variables imposed by relevant topology optimization problems, integer programming problems become far too expensive to solve. Instead, a relaxation approach is used where the variables are continuous and penalization is introduced in order to obtain a design in which the design variables are as close to 0 or 1 as possible. The penalization is such that a nonlinear behaviour of some structural property with respect to the design variable is obtained, making intermediate design variable values non-favourable and thus avoided in the final design.

The first penalization technique was introduced by Bendsøe in 1989 [4] and it is still used very frequently. This penalization technique is called SIMP, Solid Isotropic Material with Penalization, and penalizes the stiffness matrix as

$$
\boldsymbol{K}(\boldsymbol{x})=\sum_{e=1}^{m} x_{e}^{p} \boldsymbol{K}_{e},
$$

where $\boldsymbol{K}_{e} \in \mathbb{R}^{n \times n}$ is an expanded element stiffness matrix and $p>1$ a parameter that is usually set to 3 . SIMP makes the stiffness unproportionately low for intermediate design variable values. Thus, if the available mass is constrained or minimized, it is more efficient, from a stiffness point-of-view, to let the design variables approach their bounds. 


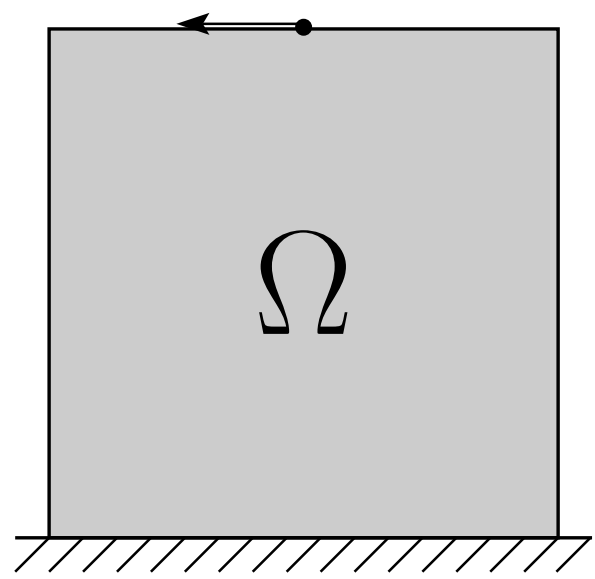

Figure 3: A typical design domain $\Omega$ and boundary conditions for a topology optimization problem.

As the stiffness matrix is required to be positive definite, a non-zero lower bound is enforced on the box constraints in $(\mathbb{P})$. These read $0<\varepsilon \leq x_{e} \leq 1$, where $\varepsilon$ is a small value. Bendsøe and Sigmund [7] recommend a typical value of about $\varepsilon=$ $10^{-3}$, meaning that an element that represents a hole has actually not zero stiffness, but $\left(10^{-3}\right)^{3}=10^{-9}$ times the element stiffness matrix. This causes numerical problems in some formulations that will be discussed later in the thesis.

No physical interpretation of what the design variables represent is given in this thesis; they are simply mathematical scale factors of element properties. As the final design should represent only solid material and voids, no physical interpretation of intermediate design variable values is required. However, several physical interpretations have been suggested in the literature, and among the most popular interpretations are element thickness in 2D-problems [45], material density [8], [10], [51], [11] or as composite material [6].

\subsection{Numerical instabilities}

The introduction of SIMP for $p>1$ makes the optimization problem non-convex. Non-convexity means not only that a minimum is not necessarily the global one, but also that small changes to the original problem, such as another starting point or changed parameter value, may result in another local optimum and thus, another optimized design. This is a known problem in topology optimization and it has not been addressed in this work.

There are also a number of other problems that arise in topology optimization. To start with, topology optimization in a continuum case may not be a well-posed 


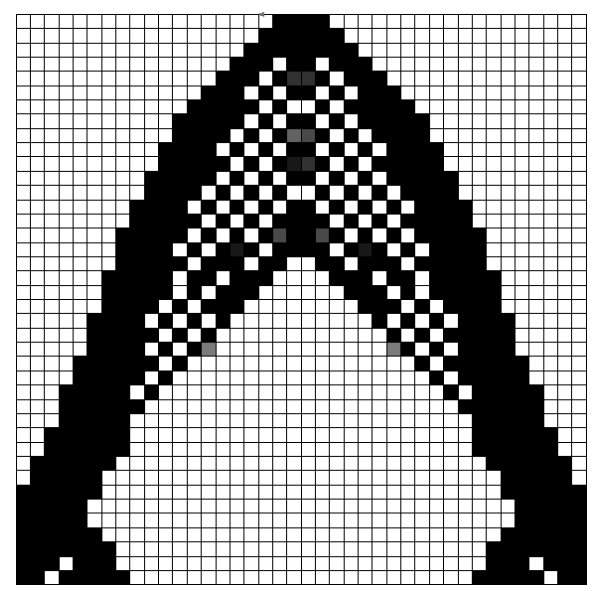

(a)

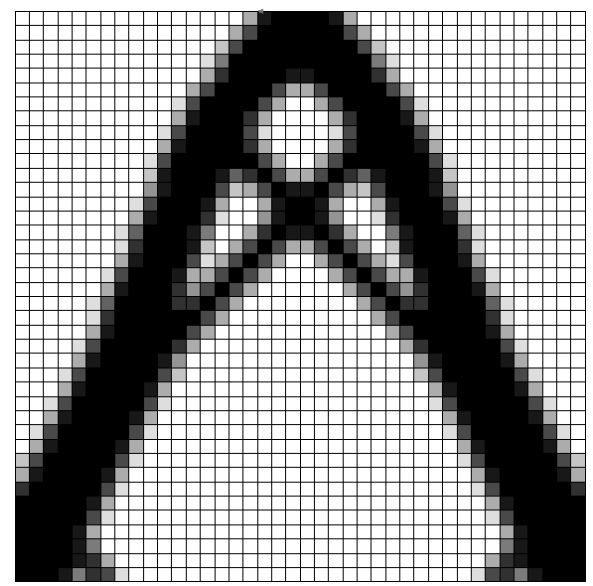

(b)

Figure 4: Compliance minimization on the domain in Figure 3. a: No filter and thus checkerboards, b: Using a design variable filter with radius 1.5 times the element size; no checkerboards appear but a (grey) transition layer between solid and void remains in the final design.

problem and no solutions may exist. This is because several thin structural members give a stiffer structure than one thick. Thus, an infinite number of infinitely thin structural members would represent the stiffest structure. This problem is to some extent avoided by the discretization, but still causes difficulties which appear as mesh-dependence, meaning that two different discretizations, one coarse and one fine, typically do not result in the same topology. A discretization with smaller elements leads to an increasing number of thinner structural parts.

Another issue is related to the fact that the optimization is made on the FE-model, not on the continuum. All weaknesses of the FE-formulation are also present in the optimization and will influence the design. One such problem, that occurs for example in models discretized by two-dimensional four-node quadrilateral elements, is that if the material distribution varies between solid and void in consecutive elements, such that the material distribution forms a checkerboard pattern, the structure becomes artificially stiff as shown by Díaz and Sigmund [21]. Such checkerboard patterns with artificial stiffness are created by the optimization. One such solution is seen in Figure 4(a), where the compliance has been minimized by distributing a limited amount of mass, i.e. with reference to $(\mathbb{P})$, $g_{0}(\boldsymbol{x})=1 / 2 \boldsymbol{f}(\boldsymbol{x})^{\top} \boldsymbol{u}(\boldsymbol{x})$ and $g_{1}(\boldsymbol{x})=\sum_{e=1}^{m} M_{e} x_{e}$, where $M_{e}$ is the mass of element $e$.

The problems due to mesh-dependency and checkerboard patterns are avoided by introducing some operator that enforces a minimum size on structural members. This is known as a filter, from the filters used in image processing [53]. 


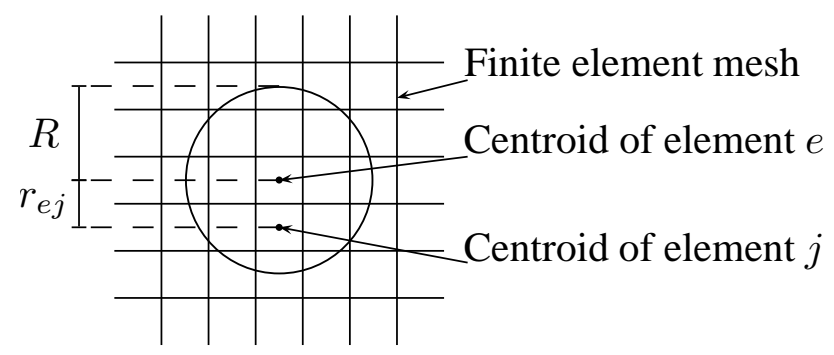

Figure 5: Visualization of a filter for design variable $x_{e}$.

\subsection{Filtering techniques}

Filters are introduced in topology optimization in order to remove checkerboard patterns and mesh dependency [55]. The first filter that was introduced was the sensitivity filter by Sigmund [51] which modifies the sensitivities (2) such that they are averaged based on the sensitivity with respect to design variables related to neighbouring elements.

Since the sensitivity filter was first published about 20 years ago it has been used extensively in different applications and has provided good solutions. Several tests with the sensitivity filter have been performed in this work, using the methods in this thesis, and they also point in this direction. However, the sensitivity filter has not been used in any of the examples shown in this thesis, because it is heuristic; when the sensitivities are modified they are no longer the correct sensitivities to the problem formulation and there is no mathematical justification of the theory. Indeed there are situations when the sensitivity filter does not perform as intended, as was noticed by Svanberg and Svärd [59].

The filter that is used in this thesis is the design variable filter (often called density filter) developed by Bruns and Tortorelli [12]. This filter is simply a change from one set of variables to another. A set of filtered variables $\rho_{e}(\boldsymbol{x})$ are created by taking a weighted average of $x_{e}$ and the values of its neighbouring elements. The filter reads

$$
\rho_{e}(\boldsymbol{x})=\sum_{j=1}^{m} \Psi_{e j} x_{j}, \quad e=1, \ldots, m,
$$

in which

$$
\Psi_{e j}=\frac{\psi_{e j} v_{j}}{\sum_{k=1}^{m} \psi_{e k} v_{k}}, \quad \psi_{e j}=\max \left(0, R-r_{e j}\right),
$$

where $v_{j}$ is the volume of element $j, R$ is the filter radius and $r_{e j}$ is the distance between the centroid of elements $e$ and $j$. See Figure 5 for a visualization.

The filtered variables $\boldsymbol{\rho}(\boldsymbol{x})$ will be referred to as physical variables, as they are used to define the properties of the structure, such as the stiffness, the stress and 
the mass. Using the SIMP-approach the stiffness matrix now reads

$$
\boldsymbol{K}(\boldsymbol{x})=\sum_{e=1}^{m} \rho_{e}(\boldsymbol{x})^{p} \boldsymbol{K}_{e} .
$$

If the filter $(8)$ is used when solving $(\mathbb{P})$ for minimum compliance with a limited amount of material, designs such as that in Figure 4(b) are obtained.

\subsection{Obtaining a black-and-white design}

A drawback with both the sensitivity filter and the design variable filter is that a grey transition layer of elements which have intermediate design variable values is created between material and void. The boundaries of the structure are therefore blurred, as in Figure 4(b), and it is not clear how the design should be interpreted. Further, due to the penalization of intermediate design variable values, the response is non-physical.

Several ways to remove this transition layer have been suggested in the literature and a comprehensive review and comparison is presented by Sigmund [53]. Most techniques rely on some differentiable approximation of minimum or maximum operators, where a minimum operator sets the design variable value to the lower bound if any element within the distance $R$ equals the lower bound, and the maximum operator does the same but with the upper bound. One such approximation is the Heaviside projection filter suggested by Guest et al. [26], where a Heaviside step function is approximated and the curvature is controlled by a parameter that is increased successively. However, the experience from studies conducted for this thesis is that the large change of the structure that occurs when the curvature parameter is updated causes difficulties when non-linear constraints, e.g. stress constraints, are present and convergence to a feasible point seems very difficult.

In this thesis a simple two-step strategy is proposed for obtaining a black-and-white design without transition layers, as proposed in paper III. First the optimization problem is solved using the design variable filter (8), then the optimization continues with modified restrictions on the design space; no filter is used and some variables are fixed. Only those design variables for which the corresponding physical variable has an intermediate value are allowed to vary in this second step. If $\rho_{e}$ is (close to) $\varepsilon$ or 1 , this means that the $x_{j}$ that are included by the filter are also (close to) $\varepsilon$ or 1 and element $e$ is thus not at a boundary, such variables are fixed to their current value. The variables in this second optimization step are defined by

$$
\boldsymbol{x}= \begin{cases}\varepsilon \leq x_{e} \leq 1 & \text { if } 2 \varepsilon<\rho_{e}^{\mathrm{opt}}<1-\varepsilon, \\ x_{e}=\rho_{e}^{\mathrm{opt}} & \text { otherwise }\end{cases}
$$

where $\boldsymbol{\rho}^{\mathrm{opt}}$ is the design obtained after convergence with the filter. The second optimization step is initialized from the physical design variable values at the end 
of the first step, and since no filter is used, $\boldsymbol{\rho}(\boldsymbol{x})=\boldsymbol{x}=\boldsymbol{\rho}^{\mathrm{opt}}$. Thus, there is no sudden change in the topology between the two steps.

There are also some obvious drawbacks with this method. Compared to using only the design variable filter, additional iterations are required before the final design is obtained and if the filter radius $R$ is much greater than the average element size, meaning that the width of the transition layer spans a high number of elements, checkerboard patterns may form in the second optimization step. However, for filter radii equal to 1.5-2.5 times the average element size, which are used in this work, no such checkerboard patterns are experienced.

\subsection{Stress singularity and penalization of stresses}

One problem when stresses are considered in topology optimization is the so-called singularity phenomenon, which is due to non-zero stresses in elements representing voids. These stresses can be so high that stress constraints are violated, thus preventing holes from being created. Of course, this stress is artificial and is a result of the mathematical modelling.

The singularity problem was first discovered on a truss design by Sved and Ginos [61] and is discussed by Kirsch [31], Cheng and Jiang [13], Rozvany and Birker [47], Guo and Cheng [27], among others.

In this work, stress singularity is avoided by using a stress penalization which has the same form as the stiffness penalization, but with an exponent $0<q<1$. The penalized stress vector,

$$
\boldsymbol{\sigma}(\boldsymbol{x})=\left(\begin{array}{llllll}
\sigma_{x}(\boldsymbol{x}) & \sigma_{y}(\boldsymbol{x}) & \sigma_{z}(\boldsymbol{x}) & \tau_{x y}(\boldsymbol{x}) & \tau_{y z}(\boldsymbol{x}) & \tau_{z x}(\boldsymbol{x})
\end{array}\right)^{\top},
$$

in the element related to design variable $\rho_{e}(\boldsymbol{x})$, is calculated as

$$
\boldsymbol{\sigma}_{e}(\boldsymbol{x})=\left(\rho_{e}(\boldsymbol{x})\right)^{q} \boldsymbol{E} \boldsymbol{B}_{e} \boldsymbol{u}(\boldsymbol{x}),
$$

where $\boldsymbol{E}$ is the constitutive matrix and $\boldsymbol{B}_{e}$ is the strain-displacement matrix corresponding to the stress evaluation point in element $e$. For notational simplicity, it is assumed that only one stress evaluation point is used for each element.

The penalization in (11) gives further penalization of intermediate design variable values, but also the desired property

$$
\lim _{\rho_{e}(\boldsymbol{x}) \rightarrow 0} \boldsymbol{\sigma}_{e}(\boldsymbol{\rho}(\boldsymbol{x}))=\mathbf{0},
$$

and thus, there is no singularity problem.

Using the stress penalization (11) and the stiffness penalization (9) gives an inconsistent relationship between stiffness and stress for intermediate design variable values. However, the penalization functions do not affect the stiffness or stress 
when a physical design variable is at its upper bound and they approach zero when the physical design variable does. As the reason for introducing the penalization is to result in a black-and-white design, the stress and stiffness in the final design will be both physical and consistent. This was used by Bruggi [10] in the so-called $q p$-approach, where the effect of different penalization exponents $q$ and $p$ were evaluated. One specific choice was $q=1 / 2$ and $p=3$ which is also used in this work. A plot of the penalization functions is shown in Figure 6 .

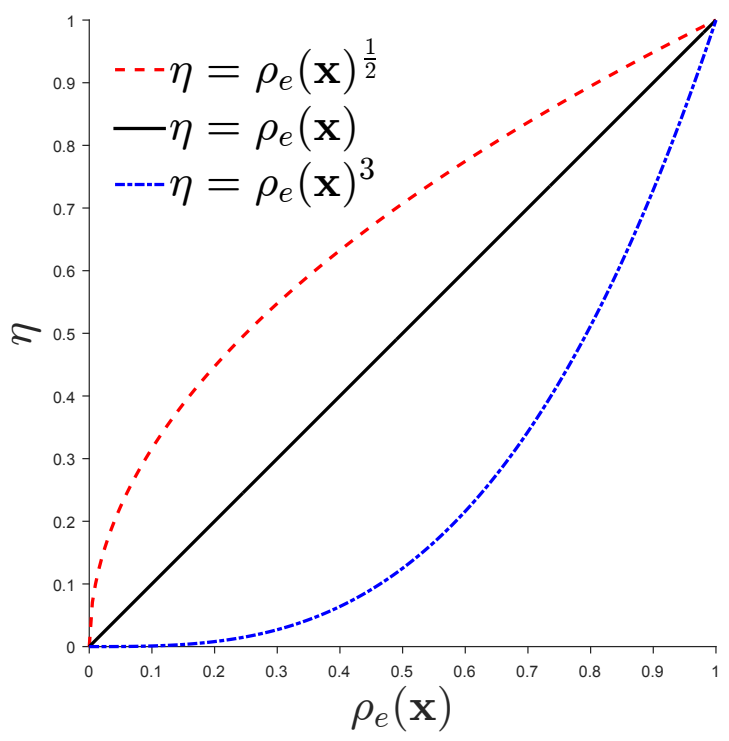

Figure 6: Penalization functions used for stress, mass and stiffness.

In the literature the SIMP penalization is often introduced as a "material" penalization, applied directly on the constitutive matrix $\boldsymbol{E}$, rather than on the stiffness matrix as in (9). Of course, in the traditional compliance formulation this gives exactly the same result as $\boldsymbol{E}$ enters linear in $\boldsymbol{K}(\boldsymbol{x})$. However, if for example stresses are included in the formulation, the same penalization is then also applied to the stresses, which has the undesired effect that the stresses are lowered for intermediate design variable values. This was noted by Duysinx and Bendsøe [23] who suggested that the stresses, calculated with a "SIMP-penalized" $\boldsymbol{E}$, should be divided by the same penalization, giving an expression similar to that in (11) but with the exponent $q=0$ (which is not allowed in this thesis). Efficiently, this means that the strain is calculated on the penalized, lower, stiffness, but that the stress is always calculated as if the element was solid. This resulted in singularity problems and this issue was circumvented by modifying the stress limit using the $\varepsilon$-relaxation approach, developed by Cheng and Guo [14], where the allowable local stress is increased as $\rho_{e}(\boldsymbol{x}) \rightarrow 0$. That is, the basic problem that stresses become high in voids remains, but the limit is scaled such that the constraint is 
not violated. The original form of the $\varepsilon$-relaxation approach also gives an increase of the allowable stress at $\rho_{e}(\boldsymbol{x})=1$, implying that the optimized design will have stresses which are too high, as discussed by Bruggi [10]. An issue with this approach is that the parameter $\varepsilon$ must be chosen appropriately and updated during the optimization. For these reasons, the stress penalization (11) is used instead of the $\varepsilon$-relaxation approach in this thesis.

\subsection{Mass penalization}

In some circumstances it might be of interest to penalize the mass, but in this work the element mass $M_{e}$ is scaled linearly with $\rho_{e}(\boldsymbol{x})$ when the mass is used as objective or constraint function.

For self-weight loaded problems another linear scaling was suggested in paper III. This scaling applies to the mass matrix when the self-weight load is calculated and reads $\left(\rho_{e}(\boldsymbol{x})-\varepsilon\right) /(1-\varepsilon)$, meaning that the self-weight load is zero when $\rho_{e}(\boldsymbol{x})=\varepsilon$, so that no self-weight load is applied in voids, and that the full load is obtained for $\rho_{e}(\boldsymbol{x})=1$. 


\section{Stress and fatigue constraints}

\subsection{On the importance of using constraints adapted to the application}

Topology optimization is traditionally used as a tool for finding optimal load paths with respect to stiffness. However, from an engineering point-of-view, few applications have maximum stiffness as the main design criterion. Usually, the design needs to have sufficient stiffness, so that buckling is avoided or the eigenfrequency is above some critical value, and the main design criteria include stress and fatigue requirements and mass minimization.

In structural optimization in general, and in topology optimization in particular, it is important to consider all major design requirements in the formulation. Otherwise, these might be very difficult to satisfy in later stages. One popular example is the minimum compliance design obtained using an L-shaped design domain, Figure 7 . The minimum compliance design creates an internal corner where the stress (in the continuum) becomes infinite, see Figures 8(a) and 8(c). Major topological changes, obtained by manual adjustments or by using shape optimization, would be required to obtain a design with reasonable stresses. Such large changes to an optimized design typically mean that the new design is far from optimal and the necessity of the initial topology optimization might be questioned. If, instead, a

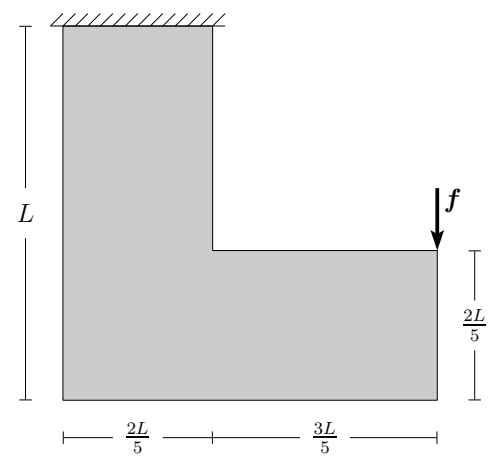

Figure 7: The L-beam problem. 


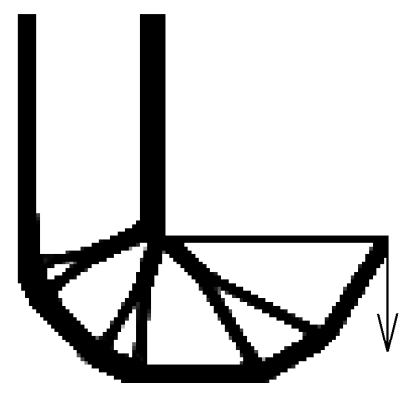

(a)

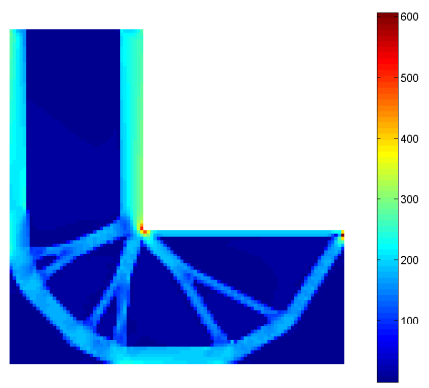

(c)

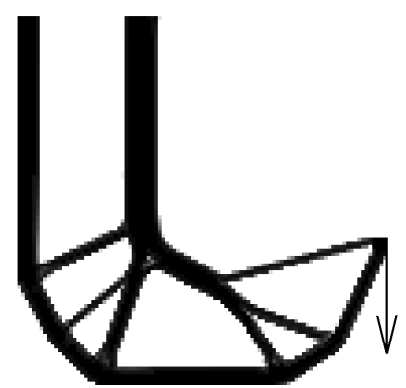

(b)

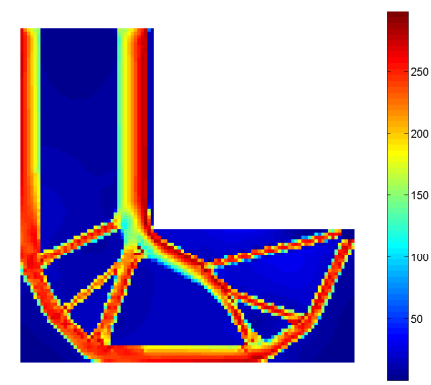

(d)

Figure 8: Optimized topologies and von Mises stresses in the final designs. (a,c): Traditional compliance formulation, $(\mathbf{b}, \mathbf{d})$ : Mass minimization problem with one stress constraint.

stress constraint is used to limit the maximum allowable stresses and the mass is minimized, the optimized topology and corresponding stress plot in Figures 8(b) and $8(\mathrm{~d})$ are obtained. This design would be a better starting point for further design work since only sizing, and no change of the topology, might be necessary.

Thus, searching for the minimum mass design that satisfies stress and fatigue life constraints is of interest for design engineers in a large variety of applications.

\subsection{Stress constraints}

Stress constraints have been discussed since the very beginning of topology optimization. For example, the pioneering works by Bendsøe and Kicuchi from 1988 [5] and by Bendsøe from 1989 [4] mention stress constraints, even though these were not used in the optimization. But it is not until quite recently that promising results have been published. 
There are mainly two problems related to stress constraints: singularity and computational cost. The singularity problem was discussed in Chapter 3.5 and is accounted for by stress penalization (11). The high computational cost is because stress is a local measure, meaning that the stress at all points in the structure need to be considered. Thus, the most correct way is to use so-called local stress constraints, where one stress constraint is used for every element (or stress evaluation point). This, however, results in $m$ number of linear systems (5) that need to be solved in each iteration and a more expensive problem for the optimization solver, making local stress constraints too expensive for anything other than very small problems.

In order to decrease the computational cost, Duysinx and Sigmund [25] formulated a global stress constraint, where there is only one stress constraint, which is formulated upon a global stress measure. The global stress measure needs to be created such that it gives a differentiable expression that is a good representation of the maximum stress in any element of the model.

A third alternative, in this work called clustered stress constraints, is where local stresses are clustered into a small number of clustered stress measures, and one stress constraint is applied to each cluster. The idea behind the clustered stress constraints is the same as that in the "block aggregated" approach by París et al. [42] or the "regional constraints" by Le et al. [33]; that is, to get a better representation of the local stresses than in the global stress constraint and at a reasonable computational cost. Clustered stress constraints were developed in paper I, where different clustering techniques and suitable clustered stress measure were evaluated, as will be discussed in Chapter 4.2.1.

Some commercial software can handle stress constraints. One of these is Optistruct [40] where the stress constraint is based on a single von Mises measure. However, the approach appears to be too rough and Optistruct finds a solution for the L-shaped beam in Figure 7 that does not avoid the singularity in the internal corner. For comparison purposes, a stress constrained topology optimization for minimum mass is first made in Optistruct and then using the methods in this work, implemented in TRINITAS [66] and using three clusters. Both models are created by four-node quadrilateral elements. The optimized designs are seen in Figure 9. The solution in Optistruct, Figure 9(a), is comparable to a compliance based design, like that in Figure 8(a), as no radius is created in the internal corner. A "minimum member size control", i.e. a filter, as described in [70], was used in Optistruct with the same radius $R$ in (8) as that used in TRINITAS. The design obtained in this work, Figure 9(b), is much lighter, about 2/3 of that obtained by Optistruct, and also creates a radius in order to avoid the stress concentration in the internal corner, where the stress will approach infinity in the design in Figure 9(a). The suggested approach for removing the stress concentrations in Optistruct [40] is to continue with local shape optimization. However, local changes will not be sufficient for the design in Figure 9(a).

The design in Figure 8(b) was obtained with another element type (eight-node 
quadrilaterals) and another load, but shows that also a global stress constraint, if formulated appropriately, is sufficient to obtain a design without the sharp internal corner.

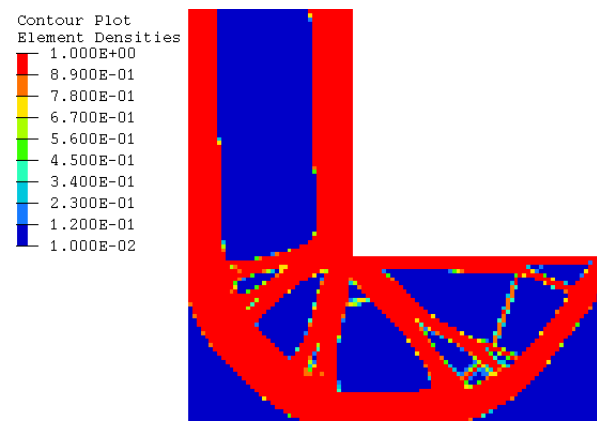

(a)

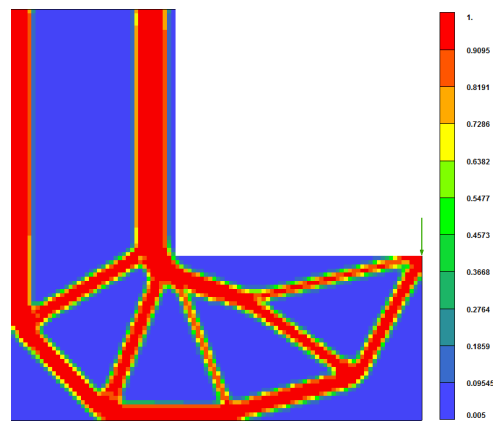

(b)

Figure 9: Comparison between commercial software and this work, for a stress constrained mass minimization problem. a: Solution obtained using Optistruct, b: Solution obtained using this work with 3 clusters.

\subsubsection{Global and clustered stress measures}

The clustered approach allows for a trade-off between accuracy and computational cost. The main reason for using clusters is to reduce the $m$ number of local constraints (one for each element) to $d \ll m$ number of clustered constraints (one for each stress cluster) and still maintain the possibility of controlling the local stresses. We may think of the two extremes, $d=1$ and $d=m$, which bring us back to the global and local approaches, respectively.

In this thesis, the local stress measure used for the stress constraints is the von Mises stress ${ }^{1}$, which in this work is based on the penalized stress vector (10). Omitting the element index and the design dependence, the von Mises stress reads

$$
\sigma^{\mathrm{vM}}=\left(\sigma_{x}^{2}+\sigma_{y}^{2}+\sigma_{z}^{2}-\sigma_{x} \sigma_{y}-\sigma_{y} \sigma_{z}-\sigma_{z} \sigma_{x}+3 \tau_{x y}^{2}+3 \tau_{y z}^{2}+3 \tau_{z x}^{2}\right)^{\frac{1}{2}} .
$$

The clustered stress measure used in this work is a modified P-norm of penalized local von Mises stresses. A P-norm has been used in earlier work, [69], [25] and [33], to group local stresses, but the modification used here is different. The clustered stress measure for cluster $i$ is defined by

$$
\sigma_{i}^{\mathrm{C}}(\boldsymbol{x})=\left(\frac{1}{N_{i}} \sum_{e \in \Omega_{i}}\left(\sigma_{e}^{\mathrm{vM}}(\boldsymbol{x})\right)^{P}\right)^{\frac{1}{P}},
$$

\footnotetext{
${ }^{1}$ The method is not restricted to von Mises stresses, e.g. principal stresses are clustered in Chapter 4.3 .
} 
where $N_{i}$ is the number of elements that belong to the set $\Omega_{i} \subset \Omega$ of elements in cluster $i$ and $P \geq 1$ is the P-norm exponent.

The clustered stress measure (12) is such that if all local stresses in the cluster are the same, i.e. $\sigma_{e}^{\mathrm{vM}}(\boldsymbol{x})=\sigma^{\mathrm{vM}}, \forall e \in \Omega_{i}$, we obtain the desired clustered stress measure

$$
\sigma_{i}^{\mathrm{C}}(\boldsymbol{x})=\left(\frac{1}{N_{i}} \sum_{e \in \Omega_{i}}\left(\sigma_{e}^{\mathrm{vM}}(\boldsymbol{x})\right)^{P}\right)^{\frac{1}{P}}=\left(\frac{1}{N_{i}}\right)^{\frac{1}{P}}\left(N_{i}\left(\sigma^{\mathrm{vM}}\right)^{P}\right)^{\frac{1}{P}}=\sigma^{\mathrm{vM}} .
$$

For all other cases, $\sigma_{i}^{\mathrm{C}}(\boldsymbol{x})$ will underestimate the local stresses, which means that the highest local stresses will not be below the stress limit. However, slightly higher local stresses can be allowed because the topology optimization is performed in a conceptual design phase, where the aim is to find a good structural shape, not to do the final sizing.

Based on the result in (13), the Stress level approach for creating the clusters, was suggested in paper I. In the stress level approach the elements are clustered such that the stresses in each cluster are as similar as possible, given $d$ number of clusters and the same number of members in each cluster. That is, the elements are not clustered on the basis of the geometric position, but on the stress level. Sorting the von Mises stresses in descending order and using the notation $\sigma_{1}^{\mathrm{vM}} \geq \sigma_{2}^{\mathrm{vM}} \geq \ldots \geq$ $\sigma_{m}^{\mathrm{vM}}$, where $\sigma_{1}^{\mathrm{vM}}$ is the highest von Mises stress in the model, the clustering scheme for the Stress level approach reads

$$
\underbrace{\sigma_{1}^{\mathrm{vM}} \geq \sigma_{2}^{\mathrm{vM}} \geq \sigma_{3}^{\mathrm{vM}} \geq \ldots . . \sigma_{\frac{m}{d}}^{\mathrm{vM}}}_{\text {cluster } 1} \geq \underbrace{\ldots \ldots \geq \sigma_{\frac{2 m}{d}}^{\mathrm{vM}}}_{\text {cluster } 2} \geq \ldots . . \sigma_{\frac{(d-1) m}{d}}^{\mathrm{vM}} \geq \underbrace{\ldots \ldots \geq \sigma_{m}^{\mathrm{vM}}}_{\text {cluster } d} .
$$

Another important issue regarding the clustering is that the clusters may have to be updated periodically. If the clusters are fixed, e.g. created on the basis of the stresses in the initial design, then after a few iterations when the stress distribution is different the points are no longer sorted into the clusters in the way that was intended. This means that the clustered stress measure may not be a good representation of the maximum local stresses. If the clusters are updated the local maximum stress representation is better, but the problem changes slightly as the clustered stress measure is calculated from a different set of points in two consecutive iterations. However, experience from paper I and paper II shows that this update is sufficiently small so that no numerical problems occur; the best result is obtained when the clusters are updated each iteration.

The exponent $P$ in (12) has a great influence on what the clustered stress measure represents: $P=1$ gives the mean stress for each cluster whereas an increasing $P$ brings the clustered stress measure closer to the maximum local stress of each cluster. As shown in [25], the limit value of (12) when $P$ approaches infinity reads

$$
\lim _{P \rightarrow \infty}\left(\frac{1}{N_{i}} \sum_{e \in \Omega_{i}}\left(\sigma_{e}^{\mathrm{vM}}(\boldsymbol{x})\right)^{P}\right)^{\frac{1}{P}}=\max _{e \in \Omega_{i}} \sigma_{e}^{\mathrm{vM}}(\boldsymbol{x}) .
$$


A large $P$ is therefore desirable, but to avoid numerical problems it should not be too large. In this work, values of $P$ between $8-24$ have been used on numerous examples without encountering any numerical problems. These values are larger than the values reported in e.g. [33] and [25] where P-norms are also used.

If a global stress constraint is used, the local stresses of which it consists will range from approximately zero (in voids) up to the highest stress. For such a large variation of local stresses, the stress measure in (12) will not be a good approximation of the highest stress. Therefore, Holmberg et al. [28] suggested and motivated numerically that for a global stress constraint, the stress measure should be created by a regular P-norm, without the $1 / N_{i}$-factor. This global stress measure was also used in paper $\mathrm{V}$, and reads

$$
\sigma^{\mathrm{G}}(\boldsymbol{x})=\left(\sum_{e=1}^{m}\left(\sigma_{e}^{\mathrm{vM}}(\boldsymbol{x})\right)^{P}\right)^{\frac{1}{P}} .
$$

The global stress measure (14) will instead overestimate the highest local stress, so that the highest stress in the final design will be lower or equal to the stress limit when a global stress constraint is used. That is, the relationship between the clustered stress measure, the highest local stress and the global stress measure can be summarized as

$$
\sigma_{i}^{\mathrm{C}}(\boldsymbol{x}) \leq \max _{1 \leq e \leq m} \sigma_{e}^{\mathrm{vM}}(\boldsymbol{x}) \leq \sigma^{\mathrm{G}}(\boldsymbol{x}), \quad i=1, \ldots, d .
$$

The design in Figure 8(b) was obtained using a global stress constraint. The stress limit was $350 \mathrm{MPa}$ and as seen in Figure 8(d), the highest local stress in the optimized design was approximately $300 \mathrm{MPa}$.

\subsection{High-cycle fatigue}

Fatigue is a structural failure that occurs due to repeated loading conditions. Highcycle fatigue implies that the number of cycles, or load repetitions, before failure is high; typically in the order of $10^{5}$ or more [49]. As fatigue is the reason for most failures it is an important design criterion and fatigue life aspects should therefore also be considered in topology optimization. The aim of introducing fatigue constraints into topology optimization in this work is not to replace a final fatigue analysis, but to find an optimized conceptual design that with the fewest changes possible can be changed into a final design, for which fatigue failure will not occur.

In this work, a traditional high-cycle fatigue methodology is used where no distinction is made between crack initiation, crack propagation and fatigue failure. The fatigue calculations have been performed using an in-house code from Saab AB [1]. Detailed descriptions of the methodology are given by e.g. Suresh [57], Schijve [49] and Dahlberg and Ekberg [19]. 
The work in this thesis focuses on structural parts of a military aircraft, for which fatigue life is often expressed in terms of flight hours. The aircraft is designed for a specific number of flight hours and a so-called safe-life approach is used, where the aircraft or part is taken out of service when the calculated life is reached. Therefore, structural optimization can be used to design a part such that fatigue will not occur during the specific finite life or before predetermined service intervals.

Fatigue constrained topology optimization is a research area that has not been explored until recently. This is to a large extent due to the problems that occur for stress constraints, but also due to the statistical and stochastic nature of fatigue analysis. Optistruct [40] has an integrated fatigue analysis software and can apply fatigue constraints in topology optimization. However, because of the performance of the stress constraint shown in Figure 9(a), the method has not been evaluated in this work.

A few authors have recently addressed topology optimization with respect to fatigue life. In paper II in this thesis, variable-amplitude loading was considered and this will be discussed in more detail below. In Svärd [60] the probability of failure for constant-amplitude loading was minimized or constrained by adapting the weakest link model by Weibull to a format similar to the P-norm (14), and a stress measure developed from that of Sines was used. The abstracts and presentations by Duysinx et al. [24] and Collet et al. [17] investigate the criteria proposed by Sines, Crossland and Goodman and perform fatigue constrained topology optimization using constant-amplitude loading. Different fatigue criteria for use in structural optimization were discussed by Mrzyglod and Zielinski in [36] and [37], and Mrzyglod used the Dang Van criterion and loading simplified to constantamplitude to perform topology optimization with an evolutionary algorithm in [34] and [35]. Desmorat and Desmorat [20] considered elasto-plastic low-cycle fatigue and maximized the fatigue life in a 3D topology optimization problem. Jeong et al. [30] applied fatigue constraints using criteria according to Goodman, Soderberg or Gerber, the response due to the alternating load was determined from a harmonic FE-analysis and the local stresses were clustered using P-norms.

\subsubsection{Load spectrum and material data}

A local load spectrum, that describes the variation of the applied load, has to be available for the fatigue analysis. The local load spectrum can be determined from a global spectrum, e.g. by the use of a global FE-model, where the global spectrum describes all the missions the aircraft is intended to fulfil during its entire life. Each mission, which for a fighter aircraft can for example be training, combat or show, is usually flown a large number of times and the loads for the manoeuvres in the missions are estimated.

Given the local load spectrum, load pairs are identified from peak and trough values using some cycle counting method, such as Rainflow count [19]. Figure 10 shows 


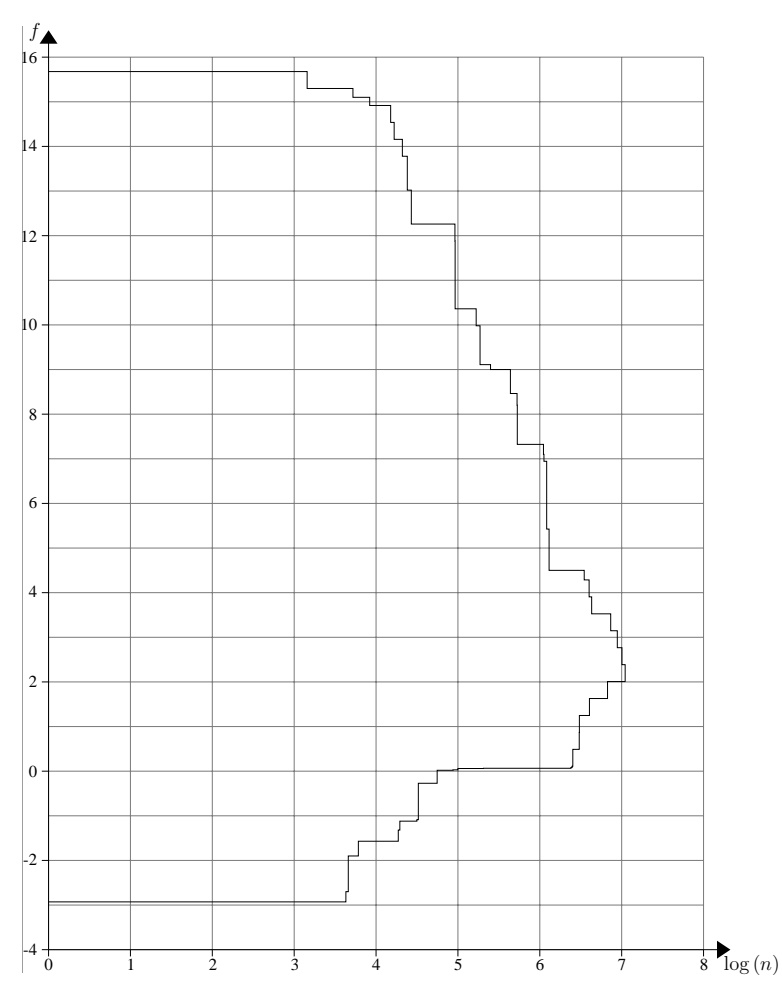

Figure 10: Load spectrum representing the load factor $f$ on the ordinate and the logarithm of the number of cycles $n$ on the abscissa.

an example of a load spectrum where load pairs have been identified. It specifies a load factor $f$, which is a multiplier of the external force $\hat{\boldsymbol{F}}$, and gives the number of cycles $n_{l}$ of each load pair $l$. The corresponding load at each load level is $\boldsymbol{f}=\hat{\boldsymbol{F}} f$.

The number of cycles allowed are determined from Wöhler- or Haigh diagrams, which are based on numerous fatigue tests made on polished test specimens. A Wöhler diagram describes the number of cycles to fatigue failure as a function of the stress amplitude for a constant load ratio $F_{\min } / F_{\max }$, where $F$ is the load in a uniaxial fatigue test. A Haigh diagram represents a series of Wöhler diagrams, obtained by varying the load ratio. It describes the allowable number of cycles $N$ for combinations between the mean stress $\sigma^{\text {mean }}=\left(\sigma_{\max }+\sigma_{\min }\right) / 2$ and the stress amplitude $\sigma^{\text {amp }}=\left(\sigma_{\max }-\sigma_{\min }\right) / 2$, as shown in Figure 11 .

Several factors influence the local resistance to crack initiation for a structural part. The loading conditions, the local stress and material properties are perhaps the most obvious and also have the greatest influence. However, the local stress might be affected by a stress concentration, which also has a prominent effect on the fatigue life. This is because the volume affected by the high stress is smaller if 


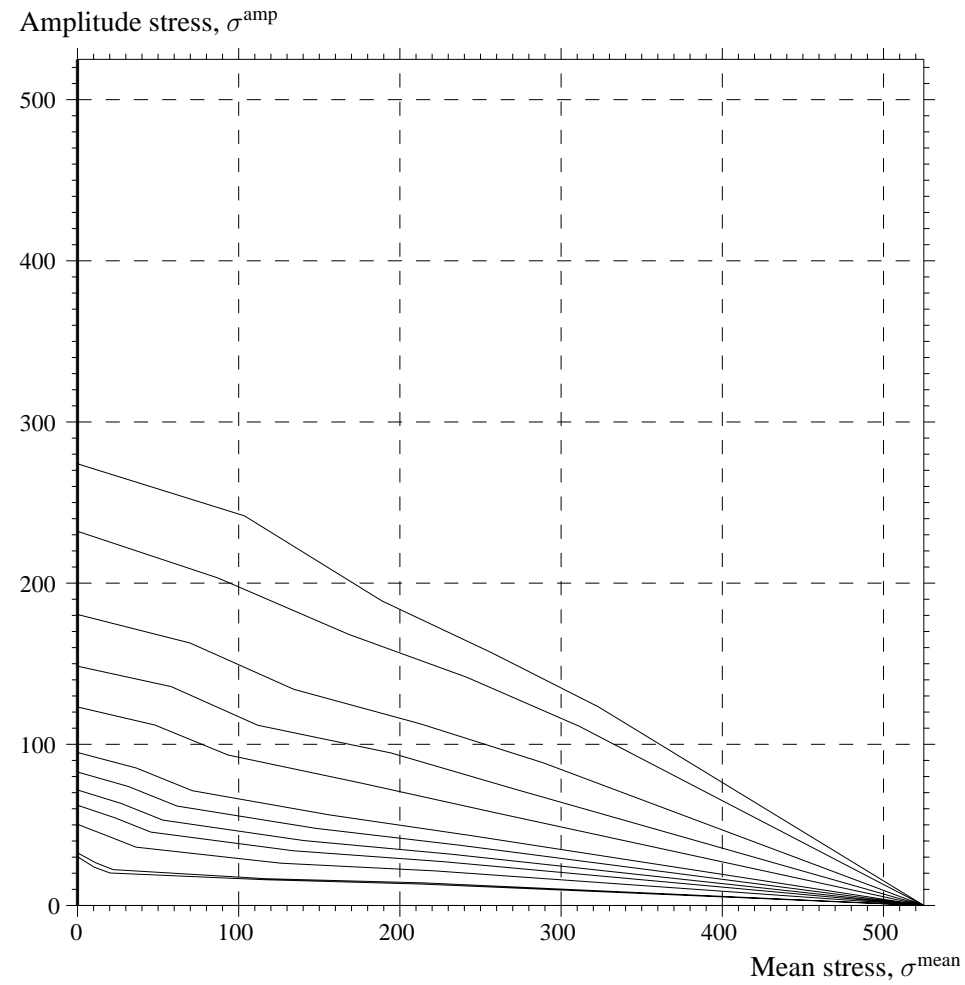

Figure 11: Haigh diagram, the curves represent constant life, i.e. different $N$.

it occurs at a stress concentration; the probability that a material defect exists in that volume is thus smaller. Using the same argument, the volume affected by a certain stress compared to the volume of the test specimen influences the expected life. The local resistance also depends on e.g. the surface roughness and surface treatment used, as well as on environmental wear. Notched test specimens are used to create Haigh diagrams for different stress concentration factors and the diagrams are constructed such that the probability of failure, based on data from the test specimens, should be below a certain percentage. The data is then reduced in order to make the diagrams valid for the specific point of interest rather than for the test specimen. Some simplifications concerning the reduction of material data are made in the optimization, as will be discussed in Chapter 4.3.3.

\subsubsection{Fatigue analysis}

In the high-cycle fatigue methodology used, the damage for each load pair in the given load spectrum is calculated and accumulated. The following describes the main steps for performing a fatigue analysis for one, pre-defined, point. 
As linear elastic material is considered, a unit load $\boldsymbol{f}_{\text {unit }}$ is used in the FE-analysis and the corresponding stress $\sigma_{\text {unit }}(\boldsymbol{x})$, at the point being analyzed, is then scaled according to the load levels in the load spectrum in order to obtain the stress at each load level as $\sigma_{\text {unit }}(\boldsymbol{x}) \hat{\boldsymbol{F}} f$.

The FE-analysis is here expressed as an operator $\mathbb{F E}$ that maps a design $\boldsymbol{x}$ and the unit load to a corresponding stress, that is

$$
\sigma_{\text {unit }}(\boldsymbol{x})=\mathbb{F} \mathbb{E}\left(\boldsymbol{f}_{\text {unit }}, \boldsymbol{x}\right) \text {. }
$$

For each load pair $l$ in the load spectrum, the corresponding mean stress $\sigma_{l}^{\text {mean }}(\boldsymbol{x})$ and amplitude stress $\sigma_{l}^{\text {amp }}(\boldsymbol{x})$ are determined by operators $\mathbb{S}_{l}$, such that

$$
\left(\sigma_{l}^{\text {mean }}(\boldsymbol{x}), \sigma_{l}^{\text {amp }}(\boldsymbol{x})\right)=\mathbb{S}_{l}\left(\sigma_{\text {unit }}(\boldsymbol{x})\right) .
$$

The number of cycles allowed for each load pair $N_{l}$ is then determined from the Haigh diagram by operators $\mathbb{H}_{l}$, as

$$
N_{l}=\mathbb{H}_{l}\left(\sigma_{l}^{\text {mean }}(\boldsymbol{x}), \sigma_{l}^{\mathrm{amp}}(\boldsymbol{x})\right)=\mathbb{H}_{l}\left(\mathbb{S}_{l}\left(\sigma_{\text {unit }}(\boldsymbol{x})\right)\right) .
$$

The cumulative damage $D\left(\sigma_{\text {unit }}(\boldsymbol{x})\right)$ is determined according to Palmgren-Miner's rule [57], by comparing the actual number of cycles $n_{l}$, given by the load spectrum, with the allowable number of cycles, $N_{l}$, for all $L$ load pairs in the spectrum. Palmgren-Miner's rule reads

$$
D\left(\sigma_{\text {unit }}(\boldsymbol{x})\right)=\sum_{l=1}^{L} \frac{n_{l}}{N_{l}}=\sum_{l=1}^{L} \frac{n_{l}}{\mathbb{H}_{l}\left(\mathbb{S}_{l}\left(\sigma_{\text {unit }}(\boldsymbol{x})\right)\right)},
$$

and fatigue failure is not expected as long as $D<1$.

\subsubsection{Fatigue constraints}

Paper II introduces high-cycle fatigue constraints, which are based on a variableamplitude load spectrum, in topology optimization. The proposed method suggests some simplifications to the fatigue analysis which allows the fatigue analysis to be separated from the topology optimization by removing the design dependence from (16)-(18). The design dependence is removed by assuming that fatigue failure will occur at a point with a certain stress concentration. Material data for this stress concentration is therefore used for the entire model and the volumetric influence is neglected. In paper II, material data for a stress concentration of 1.5 was used; experience is required to determine if this is an appropriate choice or not. If it is too conservative, the fatigue life will be underestimated, which will result in heavy structures. If it is not conservative enough, the optimization will find structures with low mass that will not satisfy the fatigue life in later design phases. The surface roughness and the surface treatment are likely to be the same for the entire 


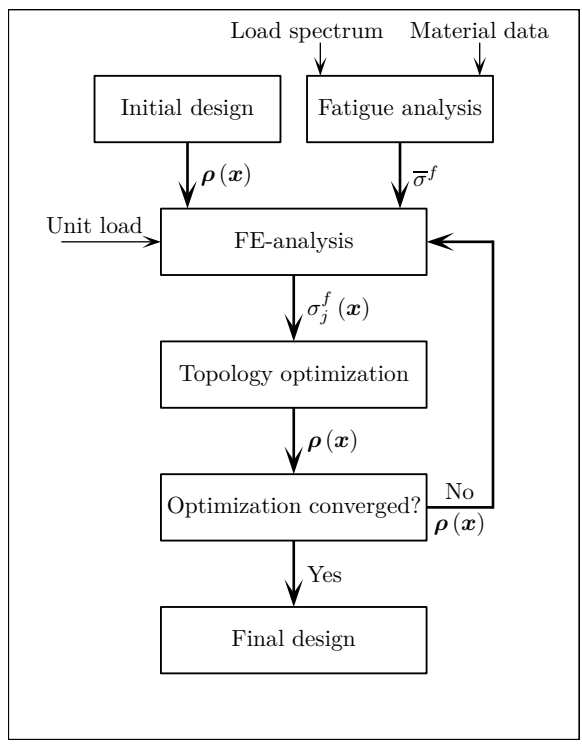

Figure 12: Flow scheme for fatigue constraints.

structure, as well as the environment it will operate in, meaning that the same reductions can be used for all points and will not change during the optimization.

The problem is thus solved in two steps. First, the critical fatigue stress $\bar{\sigma}^{f}$, which is the highest stress that gives an allowable cumulative damage $\bar{D}<1$, is found by solving the problem

$$
\left\{\begin{array}{l}
\max _{\bar{\sigma}^{f}} \bar{\sigma}^{f} \\
\text { s.t. }\left\{\sum_{l=1}^{L} \frac{n_{l}}{\mathbb{H}_{l}\left(\mathbb{S}_{l}\left(\bar{\sigma}^{f}\right)\right)} \leq \bar{D} .\right.
\end{array}\right.
$$

Then, in the second step, the critical fatigue stress is used as a constraint limit in the topology optimization, i.e. the second step is to find the design $\boldsymbol{x}$ in (15) such that the stress for the unit load does not exceed $\bar{\sigma}^{f}$. The flow scheme of the suggested methodology for fatigue constrained topology optimization is shown in Figure 12.

Fatigue is a local phenomenon, meaning that fatigue failure will occur in any point where the local resistance is not sufficient. When a fatigue analysis is made on an existing design, a relatively low number of fatigue critical spots can usually be identified and analyzed. This is not the case in topology optimization where the design is achieved iteratively. For this reason, all points need to be considered, but as for stress constraints it is not possible to use local constraints because the computational cost would be too high. Therefore, the clustered approach (12) is 


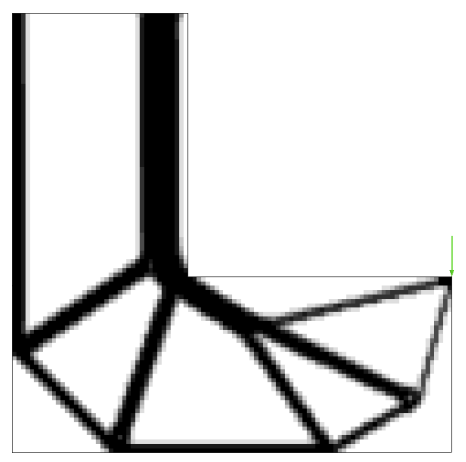

(a)

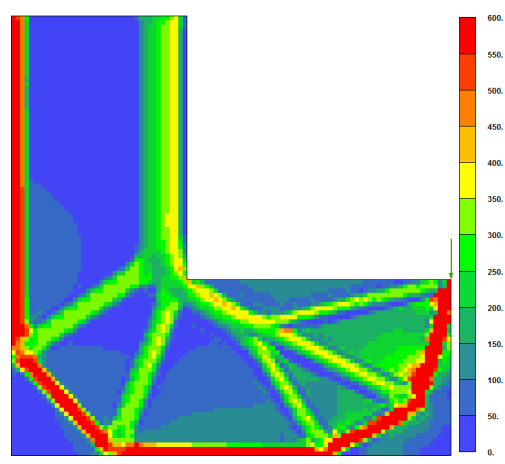

(b)

Figure 13: Minimum mass with only (tensile) highest principal stress constraints. a: Topology, b: von Mises stresses.

again used to obtain a reasonable control over the maximum local stresses at a low computational cost. The Stress level clustering technique is used and the clusters are updated every iteration. The difference is that for the fatigue constraints in this work, the highest principal stress, representing the highest tensile stress in the element, is used as the local stress measure in each element. The reason for this is that tensile stresses have a much higher influence on the fatigue life than compressive stresses and the material data used in the fatigue analysis is based on uniaxial fatigue tests; therefore the highest principal stresses correspond better to the material data than what stresses according to e.g. the von Mises criterion do. The principal stresses in a point are obtained from the eigenvalue problem

$$
\left(\left[\begin{array}{ccc}
\sigma_{x}(\boldsymbol{x}) & \tau_{x y}(\boldsymbol{x}) & \tau_{z x}(\boldsymbol{x}) \\
\tau_{x y}(\boldsymbol{x}) & \sigma_{y}(\boldsymbol{x}) & \tau_{y z}(\boldsymbol{x}) \\
\tau_{z x}(\boldsymbol{x}) & \tau_{y z}(\boldsymbol{x}) & \sigma_{z}(\boldsymbol{x})
\end{array}\right]-\lambda(\boldsymbol{x}) \boldsymbol{I}\right) \boldsymbol{\phi}(\boldsymbol{x})=\mathbf{0},
$$

in which the stress tensor is built by penalized local stresses (10), $\boldsymbol{I}$ is the identity matrix, $\lambda(\boldsymbol{x})$ are the principal stresses and $\boldsymbol{\phi}(\boldsymbol{x})$ are the eigenvectors. If the highest principal stress in element $e$ is denoted $\lambda_{e}^{1}(\boldsymbol{x})$, the clustered stress measure used for the fatigue constraints reads

$$
\sigma_{i}^{\mathrm{f}}(\boldsymbol{x})=\left(\frac{1}{N_{i}} \sum_{e \in \Omega_{i}}\left(\lambda_{e}^{1}(\boldsymbol{x})\right)^{P}\right)^{\frac{1}{P}} .
$$

If only the tensile stresses are constrained, an optimized design might have high compressive stresses, which means that a static compressive failure is to be expected. The L-shaped beam optimized for minimum mass where only tensile stresses are constrained, is shown in Figure 13 and confirms that high compressive stresses are present in the optimized design. 
For this reason, the fatigue constraints are combined with static stress constraints based on the von Mises stresses. The design in Figure 14 shows the optimized design for a mass minimization problem with seven clustered fatigue constraints and seven clustered static stress constraints. The structural members loaded in compression are now thicker than in Figure 13(a), as they are now dimensioned with respect to the allowable static stress. Notice also that the right vertical member, loaded in tension and thus sized with respect to the fatigue constraint, is slightly thicker than it was in Figure 13(a) where it was sized with respect to the static stress constraint.

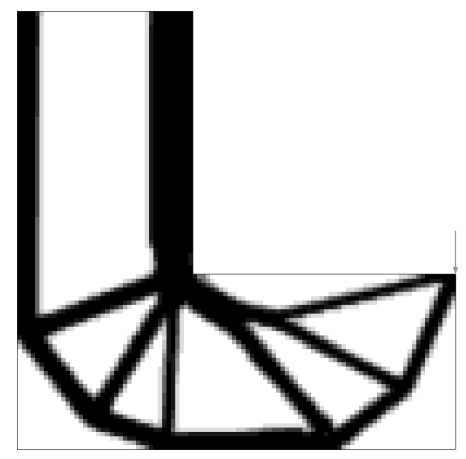

Figure 14: L-shaped beam optimized for minimum mass, with respect to fatigue life and static stress. 



\section{Optimization under load uncertainty}

One of the main issues with optimized load carrying structures is that they are typically not able to carry any other loads than those for which they were optimized. A manually designed structure will usually have some unintended robustness with respect to the loading, simply because it is not an optimal design. However, for an optimized design, a small perturbation of one of the loads might have devastating effects for the structural integrity.

In order to obtain optimized designs that are robust with respect to the loading, load uncertainties are introduced into the problem formulation and robust topology optimization is performed. In this work "robust optimization" refers to robustness with respect to loading; robustness with respect to e.g. manufacturing tolerances or material defects is not considered.

The load uncertainties might for example be formulated as small perturbations about a known load, such that the design is robust with respect to the direction of the applied load. The load uncertainty can also be a case when the loading may act in any direction in space, e.g. due to accelerations in a fighter aircraft which due to manoeuvres may have high accelerations in any direction.

Two methods to account for load uncertainties have been developed. The first formulates a semi-definite programming (SDP) framework for solving minimum compliance problems and the second solves more general problem formulations in a game theoretic framework.

\subsection{Semi-definite programming approach}

The SDP-formulation proposed in papers III and IV is based on the worst-case compliance, which is the compliance $C(\boldsymbol{x}, \boldsymbol{r})=\frac{1}{2} \boldsymbol{f}(\boldsymbol{x}, \boldsymbol{r})^{\top} \boldsymbol{u}$ for the worst loading. The loading is here described by

$$
\boldsymbol{f}(\boldsymbol{x}, \boldsymbol{r})=\boldsymbol{f}_{0}(\boldsymbol{x})+\boldsymbol{L}(\boldsymbol{x})^{\top} \boldsymbol{r},
$$

where $\boldsymbol{f}_{0}(\boldsymbol{x})$ is a fixed load and $\boldsymbol{L}(\boldsymbol{x}) \in \mathbb{R}^{s \times n}$ defines the maximum loads in all nodes and $s$ spatial dimensions. The design dependence in $\boldsymbol{f}_{0}(\boldsymbol{x})$ and $\boldsymbol{L}(\boldsymbol{x})$ is due to the possibility of considering self-weight. The uncertainty is accounted for by 
$\boldsymbol{r}$ that varies in the unit ball $T=\left\{\boldsymbol{r} \in \mathbb{R}^{s} \mid\|\boldsymbol{r}\| \leq 1\right\}$. Since the compliance is a convex function and $T$ a convex and compact set, the maximum compliance is taken at $\|\boldsymbol{r}\|=1,[44$, Corollary 32.3.1]. Thus, the worst-case compliance reads

$$
P(\boldsymbol{x})=\max _{\|\boldsymbol{r}\|=1} C(\boldsymbol{x}, \boldsymbol{r})=\max _{\|\boldsymbol{r}\|=1} \frac{1}{2} \boldsymbol{f}(\boldsymbol{x}, \boldsymbol{r})^{\top} \boldsymbol{K}(\boldsymbol{x})^{-1} \boldsymbol{f}(\boldsymbol{x}, \boldsymbol{r}),
$$

where (1) is used in the last step.

The optimization problem is now formulated as minimization of the worst-case compliance for a given mass $\bar{M}$ :

$$
\left\{\begin{array}{l}
\min _{\boldsymbol{x} \in \mathbb{R}^{m}} P(\boldsymbol{x}) \\
\text { s.t. }\left\{\begin{array}{l}
\sum_{e=1}^{m} M_{e} \rho_{e}(\boldsymbol{x})=\bar{M} \\
\varepsilon \leq x_{e} \leq 1, e=1, \ldots, m .
\end{array}\right.
\end{array}\right.
$$

A standard procedure to solve a minmax problem such as (21) is to rewrite it as a bound formulation using the additional variable $z$, varying in the non-negative set $\mathbb{R}_{+}$. The bound formulation reads

$$
\left\{\begin{array}{l}
\min _{\boldsymbol{x} \in \mathbb{R}^{m}, z \in \mathbb{R}_{+}} z \\
\text { s.t. }\left\{\begin{array}{l}
\boldsymbol{f}(\boldsymbol{x}, \boldsymbol{r})^{\top} \boldsymbol{K}(\boldsymbol{x})^{-1} \boldsymbol{f}(\boldsymbol{x}, \boldsymbol{r}) \leq z, \forall \boldsymbol{r} \in T, \\
\sum_{e=1}^{m} M_{e} \rho_{e}(\boldsymbol{x})=\bar{M} \\
\varepsilon \leq x_{e} \leq 1, e=1, \ldots, m .
\end{array}\right.
\end{array}\right.
$$

As is shown in papers III and IV, (22) may be replaced by the semi-definite programming problem

$$
\left\{\begin{array}{l}
\min _{\boldsymbol{x} \in \mathbb{R}^{m}, z \in \mathbb{R}_{+}, \mu \in \mathbb{R}_{+}} z \\
\text { s.t. }\left\{\begin{array}{l}
\left(\begin{array}{cc}
\mu \boldsymbol{I} & \mathbf{0} \\
\mathbf{0} & z-\mu
\end{array}\right)-\left(\begin{array}{c}
\boldsymbol{L}(\boldsymbol{x}) \\
\boldsymbol{f}_{0}(\boldsymbol{x})^{\top}
\end{array}\right) \boldsymbol{K}(\boldsymbol{x})^{-1}\left(\boldsymbol{L}(\boldsymbol{x})^{\top} \boldsymbol{f}_{0}(\boldsymbol{x})\right) \succeq \mathbf{0}, \\
\sum_{e=1}^{m} M_{e} \rho_{e}(\boldsymbol{x})=\bar{M} \\
\varepsilon \leq x_{e} \leq 1, e=1, \ldots, m
\end{array}\right.
\end{array}\right.
$$

where $\succeq \mathbf{0}$ means positive semi-definite and $\mu$ is an additional variable.

Numerically, (23) is solved as an ordinary non-linear optimization problem by noting that a symmetric positive semi-definite matrix $\boldsymbol{A}$ can be written $\boldsymbol{A}=\boldsymbol{C C}^{\top}$, where $\boldsymbol{C}$ is a Cholesky factor. By treating the components of the Cholesky factor as variables, varying in the set of lower triangular matrices with non-negative 
diagonal entries $\mathcal{L}_{+}^{s+1}$, a final form of the problem may be written

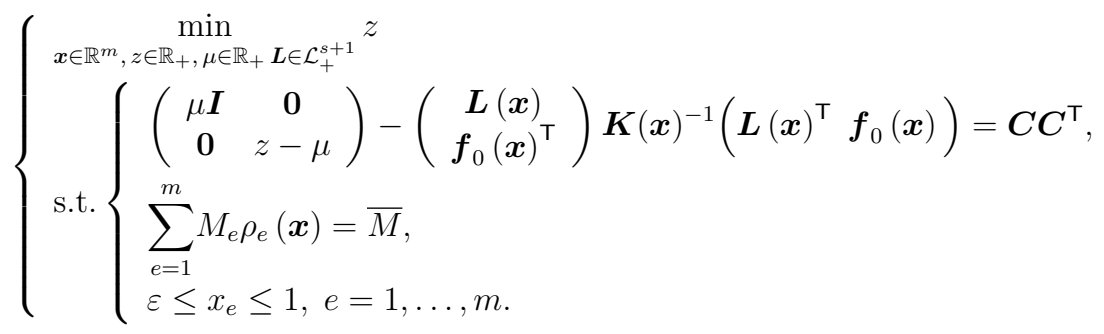

The matrix equality constraint in $(24)$ is of small size $(s+1) \times(s+1)$, and can be simplified to size $s \times s$ if no fixed loading is present, i.e. if $\boldsymbol{f}_{0}=\mathbf{0}$. This makes $(24)$ efficient for solving large scale problems. Indeed, the main computational cost in (24) is due to $\boldsymbol{K}(\boldsymbol{x})^{-1}\left(\boldsymbol{L}(\boldsymbol{x})^{\top} \boldsymbol{f}_{0}(\boldsymbol{x})\right)$, which is evaluated by solving the linear systems

$$
\boldsymbol{K}(\boldsymbol{x}) \boldsymbol{R}=\left(\boldsymbol{L}(\boldsymbol{x})^{\top} \boldsymbol{f}_{0}(\boldsymbol{x})\right)
$$

for $\boldsymbol{R} \in \mathbb{R}^{n \times(s+1)}$, meaning that the computational cost for taking all loads defined by (19) with $\boldsymbol{r} \in T$ into account, can be compared to solving (1) for $s+1$ load cases.

In paper III, a hemisphere design space, shown in Figure 15(a), is discretized by 327493 four-node tetrahedral elements. The structure is allowed to occupy $5 \%$ of the hemisphere, which is rigidly attached at the flat surface and has an attached point mass that is 10 times heavier than the final structure. The uncertain loading is due to an uncertain acceleration of the applied point mass and the mass of the structure. If the acceleration is allowed to act with the same magnitude in any direction, the optimized design is the tripod structure in Figures 15(b), 15(c) and $15(d)$.

In paper IV, a cylinder is discretized by 14640 eight-node hexahedral elements and a fixed load acts in the negative $z$-direction. There is also an uncertain load, applied at the same point and acting in any direction, which gives a resultant load varying as shown in Figure 16(a). The optimized topology is seen in Figure 16(b) and is intuitively a more robust design than that in Figure 16(c), which is a non-robust design obtained by applying only the fixed load.

\subsubsection{Comparison with eigenvalue formulation}

Worst-case compliance problems have previously been solved using an eigenvalue formulation, $[9,16,63]$. If $\boldsymbol{f}_{0}=\mathbf{0}$ in (19), a similar formulation may be obtained from (20). Introducing the notation $\boldsymbol{H}(\boldsymbol{x})=\boldsymbol{L}(\boldsymbol{x}) \boldsymbol{K}(\boldsymbol{x})^{-1} \boldsymbol{L}(\boldsymbol{x})^{\top}$, (20) may be written

$$
\max _{\|\boldsymbol{r}\|=1} \frac{1}{2} \boldsymbol{r}^{\top} \boldsymbol{H}(\boldsymbol{x}) \boldsymbol{r}=\frac{1}{2} \lambda_{1}(\boldsymbol{H}(\boldsymbol{x}))
$$




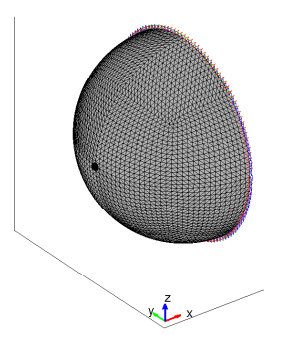

(a)

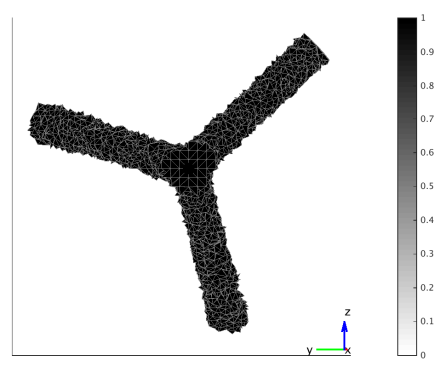

(c)

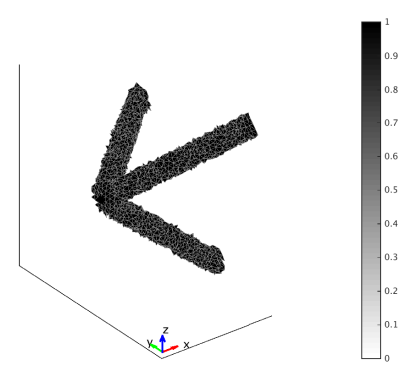

(b)

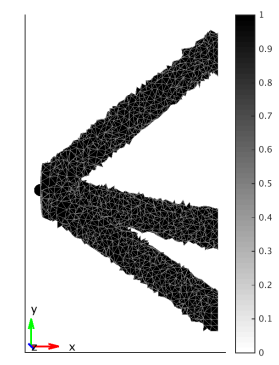

(d)

Figure 15: Hemisphere. a: Mesh, boundary conditions and point mass, b,c,d: Robust optimized design, isometric view, top view and side view.

where the equality follows from the Rayleigh-Ritz theorem ([29, Theorem 4.2.2]) and the eigenvalues $\lambda_{i}(\boldsymbol{H}(\boldsymbol{x})), i=1, \ldots, s$, of the $s \times s$ matrix $\boldsymbol{H}(\boldsymbol{x})$ are ordered such that $\lambda_{1} \geq \lambda_{2}\left(\geq \lambda_{3}\right.$ in $\left.3 \mathrm{D}\right)$. The problem with the eigenvalue formulation is that if $\lambda_{1}(\boldsymbol{H}(\boldsymbol{x}))$ is a multiple eigenvalue, i.e. if $\lambda_{1}(\boldsymbol{H}(\boldsymbol{x}))=\lambda_{2}(\boldsymbol{H}(\boldsymbol{x}))$, the derivatives are not well defined and only so-called directional derivatives, see [50], [43] or [41, Theorem 4], can be obtained. As shown numerically in paper III and repeated in the following example, multiple eigenvalues are likely to be present, not only in the solution, but also in iterations towards a solution.

In Figure 17, the L-shaped domain is considered again. The point mass, visualized as a black dot in Figure 17(a), has the same mass as the final structure. The loading is due to accelerations of the point mass and the mass of the structure and is restricted to an elliptical region, as the acceleration in the $x$-direction is twice that in the $y$-direction. The robust worst-case compliance design is seen in Figure 17(c) and it is compared to a non-robust design, Figure 17(d), optimized for two load cases where the magnitudes of the loads are defined by the ellipse and the orientations are in the $x$ and $y$-direction, respectively. 


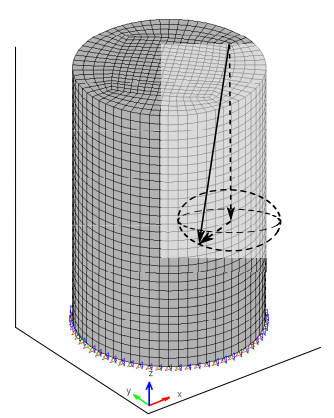

(a)

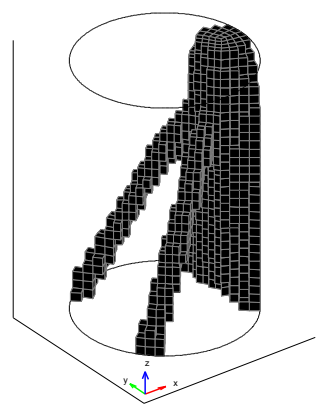

(b)

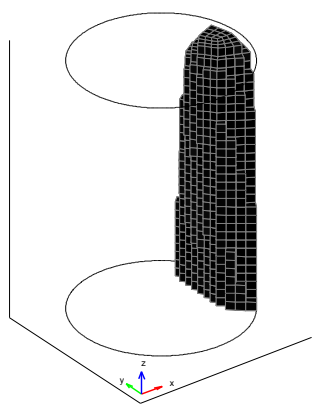

(c)

Figure 16: Cylinder with non-centered load. a: Mesh, fixed displacements, fixed load (dashed arrow in negative $z$-direction) and uncertain load (dashed arrow inside the sphere), b: Robust worst-case compliance design, c: Non-robust minimum compliance design for the fixed load only.

The plots in Figure 17(e) are obtained by plotting the compliance for different loads which vary in a counter-clockwise manner on the elliptical region, obtained by choosing $\boldsymbol{r}=(\cos \theta, \sin \theta)^{\top}, \theta \in[0,2 \pi]$. The compliance for the non-robust design is varying, i.e. there exists one "worst" and one "best" direction for the loading and the worst direction for the optimized design was not one of those for which it was optimized. For the robust design, the compliance is the same for all allowable loads, which implies robustness. That the compliance is the same for all loads also implies that the eigenvalue formulation (25) would give multiple eigenvalues. To show that multiple eigenvalues are also present in iterations towards the solution, the eigenvalue problem has been solved in each iteration in order to create the plot in Figure 17(b) which shows the highest and lowest eigenvalue for each iteration. In this example, multiple eigenvalues were obtained at iteration 20 and the eigenvalues remained multiple throughout the entire optimization.

\subsection{Game theory approach}

Game theory is a mathematical form of decision making for players that cooperate or antagonize in order to maximize their profit (or minimize loss). Each player has a pay-off function which is influenced by the strategy chosen. Usually, there is a limited amount of resources, meaning that one player's gain is another player's loss. A comprehensive introduction to game theory is given by Aubin [3].

Paper V suggests that robust topology optimization with uncertain loading can be solved as a two-player non-cooperate game formulated on the form $\mathbb{P}$, where the optimization variables are the strategies and two objective functions are used. The first player controls the structure using the standard topology optimization 


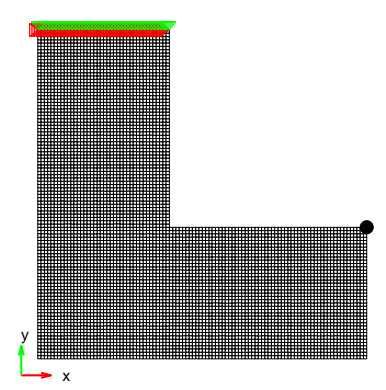

(a)

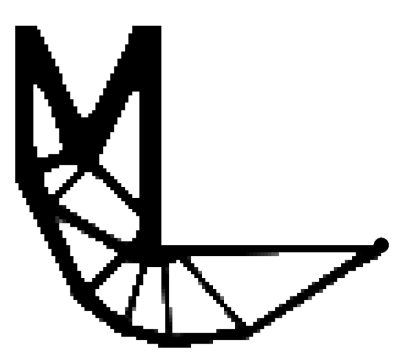

(c)

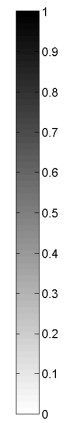

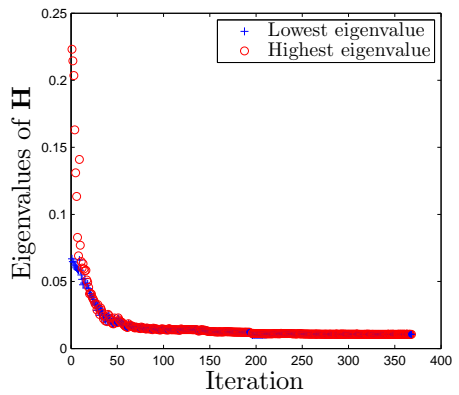

(b)

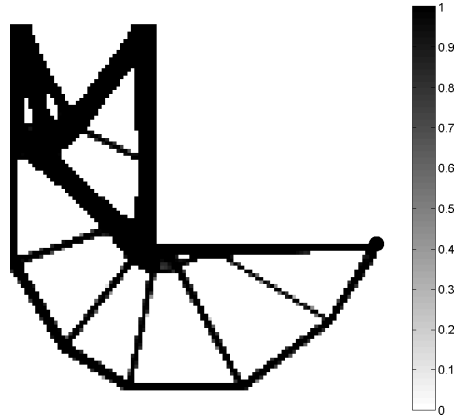

(d)

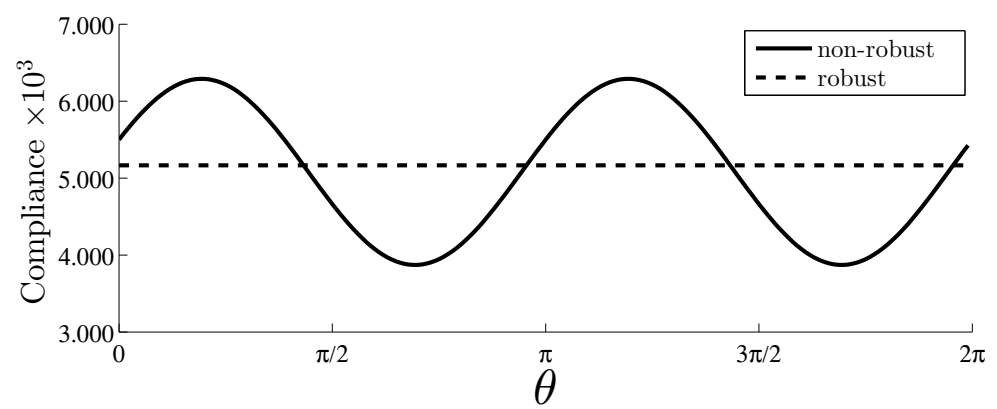

(e)

Figure 17: L-shaped beam subjected to accelerations in any direction in the plane, the magnitude is defined by an ellipse where the maximum acceleration in $x$ direction is twice of that in $y$-direction. a: Mesh, boundary conditions and point mass, b: Convergence history of the eigenvalues of $\boldsymbol{H}(\boldsymbol{x})$ for the design in Figure $17(\mathrm{c})$, the eigenvalues are multiple from iteration 20, c: Robust design, $\mathbf{d}$ : Nonrobust design obtained for two load cases, one load in $x$ and one in $y$-direction, e: Compliance, for the optimized designs in Figure 17(c) and Figure 17(d), as a function of loads varying counter-clockwise on the elliptical loading region using $\boldsymbol{r}=(\cos \theta, \sin \theta)^{\top}, \theta \in[0,2 \pi]$. 
variables $\boldsymbol{x}$ and the second player controls the load using the variables $\boldsymbol{\theta} \in \mathbb{R}^{s-1}$, which are the angles in a polar or spherical coordinate system, for $2 \mathrm{D}$ and $3 \mathrm{D}$ problems respectively, which parameterize $\boldsymbol{r}$ in (19) as shown in Figure 18(a). The force now reads

$$
\boldsymbol{f}(\boldsymbol{x}, \boldsymbol{r}(\boldsymbol{\theta}))=\boldsymbol{f}(\boldsymbol{x}, \boldsymbol{\theta})=\boldsymbol{f}_{0}(\boldsymbol{x})+\boldsymbol{L}(\boldsymbol{x})^{\top} \boldsymbol{r}(\boldsymbol{\theta}),
$$

where $\boldsymbol{L}(\boldsymbol{x})$, as already mentioned, is built by the maximum nodal loads in the global coordinate directions, here denoted $x_{\max }, y_{\max }$ and $z_{\max }$, respectively, for a single point load used in this section. For a 3D-problem $\boldsymbol{r}(\boldsymbol{\theta})$ reads

$$
\boldsymbol{r}(\boldsymbol{\theta})=\left\{\begin{array}{c}
\sin (\phi) \cos (\theta) \\
\sin (\phi) \sin (\theta) \\
\cos (\phi)
\end{array}\right\}
$$

and it simplifies to a polar system for $\phi=\pi / 2$ in 2D. An example of how the uncertain load $\boldsymbol{L}(\boldsymbol{x})^{\top} \boldsymbol{r}(\boldsymbol{\theta})$ varies in a 2D-problem is shown in Figure 18(b) for the case when $x_{\max }=2 y_{\max }$.

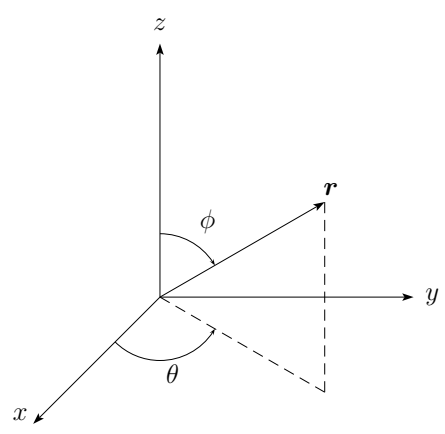

(a)

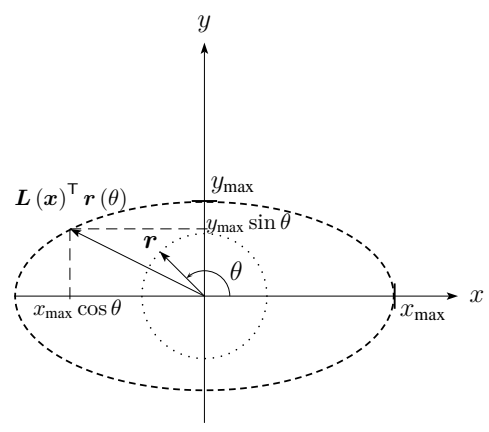

(b)

Figure 18: Visualization of how the uncertainty vector and the uncertain load is defined by $\boldsymbol{\theta}$. a: Spherical coordinate system used in 3D, which simplifies to a polar system for $\phi=\pi / 2$, b: Uncertain loading in 2D.

The concept of a solution is not as obvious as in standard optimization problems. In this work a solution $\left(\boldsymbol{x}^{*}, \boldsymbol{\theta}^{*}\right)$ is defined as a Nash equilibrium point [39] which defines a situation where neither of the players has an incentive to change strategy. If player one wants to minimize the objective function $g_{0}^{(1)}(\boldsymbol{x}, \boldsymbol{\theta})$ and the second player wants to maximize the objective function $g_{0}^{(2)}(\boldsymbol{x}, \boldsymbol{\theta})$, the game theoretic 
problem may be formulated as finding $\boldsymbol{x}^{*} \in \mathbb{R}^{m}$ and $\boldsymbol{\theta}^{*} \in \mathbb{R}^{s-1}$, such that

$$
\left\{\begin{array}{l}
\min _{\boldsymbol{x} \in \mathbb{R}^{m}} g_{0}^{(1)}\left(\boldsymbol{x}, \boldsymbol{\theta}^{*}\right) \\
\text { s.t. }\left\{\begin{array}{l}
g_{i}\left(\boldsymbol{x}, \boldsymbol{\theta}^{*}\right) \leq \bar{g}_{i}, i=1, \ldots, c, \\
\varepsilon \leq x_{e} \leq 1, e=1, \ldots, m,
\end{array}\right. \\
\max _{\boldsymbol{\theta} \in \mathbb{R}^{s-1}} g_{0}^{(2)}\left(\boldsymbol{x}^{*}, \boldsymbol{\theta}\right) \\
\text { s.t. } \quad \underline{\theta}_{e} \leq \theta_{e} \leq \bar{\theta}_{e}, e=1, \ldots, s-1 .
\end{array}\right.
$$

A visualization of a Nash equilibrium point for an unconstrained problem with only one variable for each player and with simple objective functions, is given in Figure 19. The Nash equilibrium point $\left(x^{*}, \theta^{*}\right)$ is the point where $\partial g_{0}^{(1)}\left(x^{*}, \theta^{*}\right) / \partial x=0$ and $\partial g_{0}^{(2)}\left(x^{*}, \theta^{*}\right) / \partial \theta=0$.

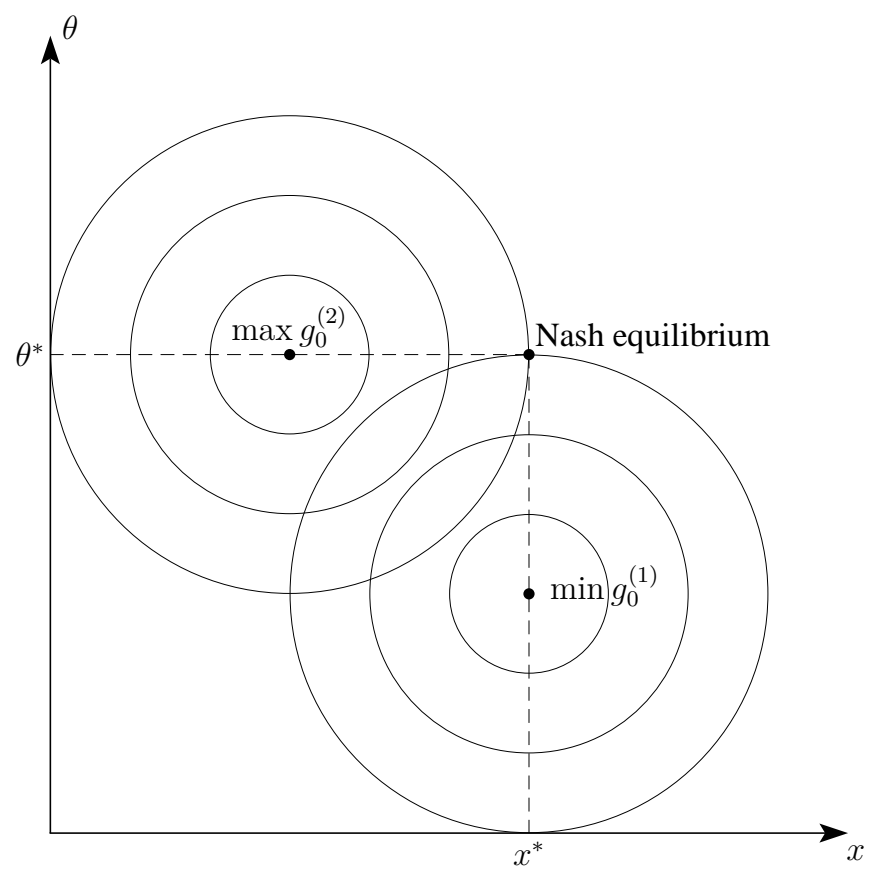

Figure 19: Visualization of a Nash equilibrium point for an unconstrained problem with only one variable per player, the circles represent level surfaces of the functions $g_{0}^{(1)}(x, \theta)$ and $g_{0}^{(2)}(x, \theta)$.

If both $g_{0}^{(1)}(\boldsymbol{x}, \boldsymbol{\theta})$ and $g_{0}^{(2)}(\boldsymbol{x}, \boldsymbol{\theta})$ are chosen as the compliance, the problem is in principle the same type as that solved using (24). However, the game theoretic 
framework presented here is more general, because it can be used to formulate problems based on any differentiable functions $g_{0}^{(1)}(\boldsymbol{x}, \boldsymbol{\theta})$ and $g_{0}^{(2)}(\boldsymbol{x}, \boldsymbol{\theta})$.

A simple algorithm is used where the load and topology problems are solved in sequence. In paper $\mathrm{V}$ it is discussed that the nature of the suggested games is such that they are difficult to converge; only in special cases does there exist one specific "worst" load that remains the worst when the topology is changed. These convergence problems are limited by adding a small number of load cases in the topology problem.

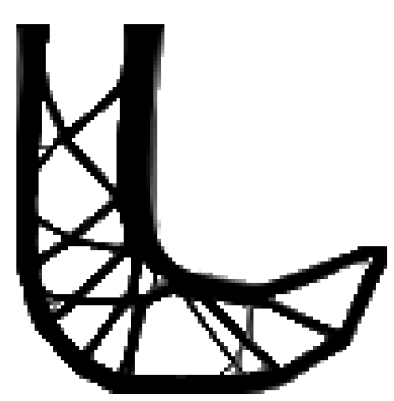

(a)

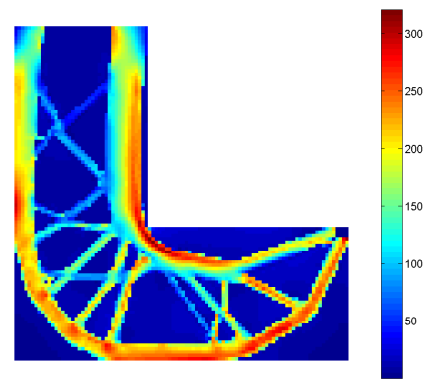

(c)

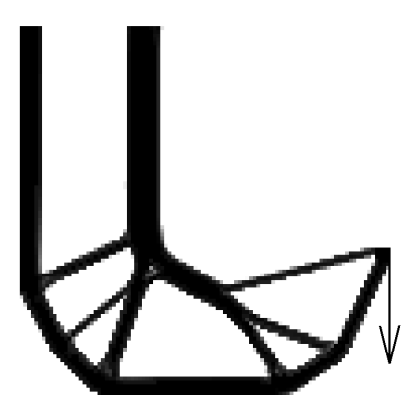

(b)

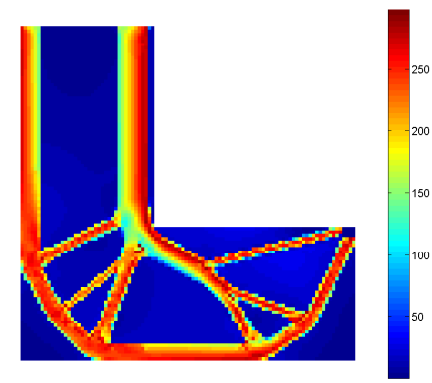

(d)

Figure 20: L-beam design. a: Robust design, b: Non-robust design with only one fixed load (as shown by the arrow), c: von Mises stresses in the robust design for the load defined by $\theta^{*}, \mathbf{d}$ : von Mises stresses in the non-robust design.

In paper $\mathrm{V}$ three different games are formulated based on the responses: compliance; global stress; and mass. For example, the mass is minimized under a global stress constraint using (14) and the loading is chosen such that the global stress is maximized. Figures 20(a) and 20(c) show the result from such a game solved on the L-shaped geometry for a load that may act in any direction in the plane and Figures 20(b) and 20(d) show a non-robust design which is the result of a standard, one load case, topology optimization problem. As can be expected, more structural members are created for the robust design in order to keep the stresses below the 
stress limit $350 \mathrm{MPa}$ for all possible loads.

The same game is also solved on the 3D-geometry in Figure 21(a) and the result is seen in Figures 21(b)-21(c). The maximum stress in Figure 21(c) is close to the stress limit and all parts should be close to the stress limit for some $\boldsymbol{\theta}$. The few grey structural members in the optimized design are explained by the discretization; a finer discretization would be required to obtain a more black-and-white design, because some structural parts cannot be made thinner without being removed.

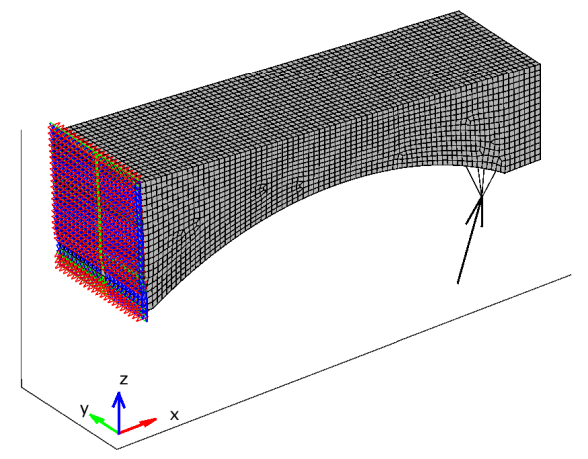

(a)

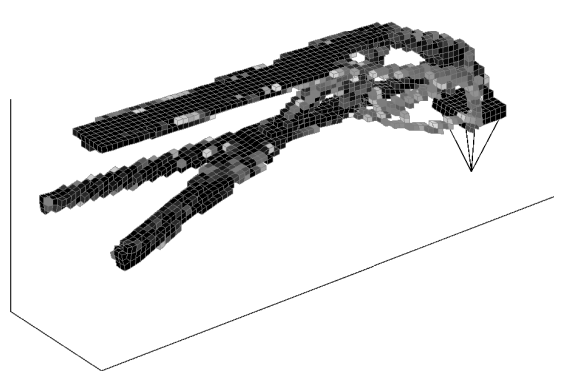

(b)

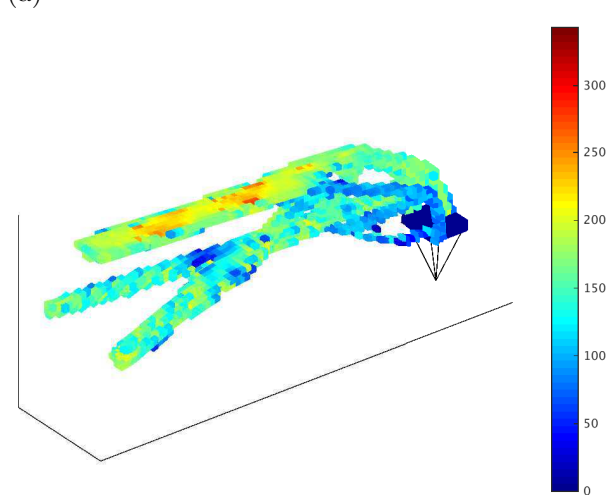

(c)

Figure 21: 3D-bracket. a: Mesh, fixed displacements and the applied (uncertain) load, b: Robust design, $\mathbf{c}$ : von Mises stresses for the load defined by $\boldsymbol{\theta}^{*}$. 


\section{Software}

Two different software have been used for the optimizations during this work. The methods in papers I and II were implemented in TRINITAS [66], while papers III-V used a Matlab-based finite element and topology optimization program that has been developed within this project.

\subsection{Developed FEM and optimization program}

A finite element and topology optimization program has been developed in Matlab [64]. From the very beginning, the main idea of the program has been that it reads in-data files of FE-models created in commercial pre-processing programs and that it is written in a general manner, so that it is easy to add e.g. new element types and objective or constraint functions. The in-data file format of choice is that used in Optistruct [40] and models can thus be prepared in e.g. the commercial pre-processing program HyperMesh [2]. This means that CAD-models can be imported and that modern mesh techniques can be used to create the FE-models. Additionally, it allows for comparisons with commercial optimization software.

The Matlab program handles several element types and different variations of the elements, such as lower order and higher order formulations and various integration schemes. The current element library consists of quadrilateral and triangular elements in 2D and hexahedral, tetrahedral, pentahedral and pyramid elements in 3D, as well as rods and mass elements. Shell elements, based on 8-node quadrilaterals, are also implemented but so far with some limitations.

The optimization problem is defined via a graphical user interface (GUI), see Figure 22 , where a browser is used to select the in-data file and a number of options and settings are available. Objective functions and constraint functions can be combined by the user, and among the functions that are available and published in research papers, are von Mises stress, principal stress, compliance, mass and mass fraction. Other functions that are available, but not tested sufficiently or used in a publication, include eigenfrequency and buckling.

Several filtering techniques are implemented, such as the design variable filter (8) developed by Bruns and Tortorelli [12] as well as a modification of this filter to create more black and white designs, see Chapter 3.4 or paper III. The derivative 


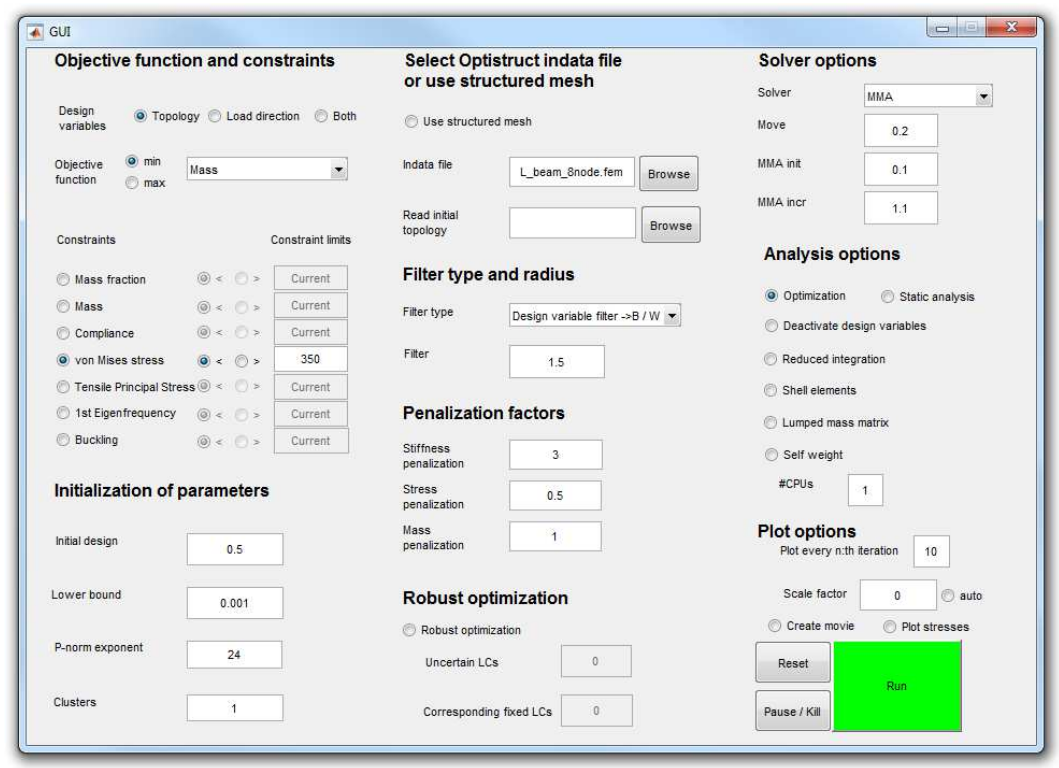

Figure 22: The GUI that selects an indata file, sets up the optimization problem and initiates the program.

filter by Sigmund [51] and dilate and erode filters using Heaviside and modified Heaviside functions $[26,53]$ are also available.

For solving the linear systems, such as the state equation (1) and systems arising in the gradient calculation (5), the Matlab function mldivide (backslash operator) is used if the available memory is sufficient. Depending on the input, this function chooses among different direct solver algorithms in order to minimize the computational time. For larger problems where the available memory becomes an issue, a preconditioned conjugate gradient method is used instead. The preconditioner is an incomplete Cholesky factorization of the coefficient matrix and the scaling introduced in [68] is also used in order to decrease the condition number of the coefficient matrix.

The optimization problem is solved using the Method of Moving Asymptotes [58], IPOPT [67] or the optimization solver in Matlab, fmincon. The minimum compliance problem can also be solved using the optimality criteria algorithm [7].

Robustness with respect to load uncertainties can for stiffness based problems be solved using the semi-definite program (24), using the eigenvalue formulation (25), or using game theory. The game theory formulation is more general as is discussed in Chapter 5.2, and can involve any of the implemented objective and constraint functions. An optimization problem may also be defined using only the load variables in order to find the worst load direction for an FE-model when 
performing a stress analysis, for example.

From a user point-of-view, a semi-definite programming problem is formulated on the standard form $(\mathbb{P})$ from the GUI, where "Robust optimization" is chosen and the uncertain and corresponding fixed loads are defined using the identification numbers assigned in the pre-processor. The program then formulates the problem (23), which is then treated by an interface to fminsdp [65], in which it is reformulated into the standard non-linear optimization problem in (24).

The initial design is an equal distribution using a value chosen in the GUI, or a design from a previously saved result. Non-design elements, which are part of the structural analysis but are not assigned a design variable, may be defined from element sets created in the pre-processor.

So-called superelements [40] can be imported and used in the optimization. Superelements represent stiffness and loads from structural parts that have been reduced from the model. The number of elements in the model can therefore be reduced significantly and replaced by direct input to the degrees of freedom related to the interface nodes. Boundary conditions with correct stiffness and loads can thus be used at a very low computational cost. The example in the following chapter also uses superelements to be able to include rigid body elements that are not implemented in the Matlab program.

The topology during the iterations can be plotted in Matlab, together with the stresses (von Mises or principal stresses) and the convergence history. The plots can be made on the nominal, undeformed shape, or using the displacements or mode shapes to plot a deformed shape. The discretized design domain with the boundary conditions is plotted once and gradients can also be plotted as a contour plot on the topology, which may be useful during development.

The final design is saved to an indata-file in Optistruct format. This file can be opened in several commercial software that offer better visualization of 3D-designs and it also facilitates when distributing the optimization result to others. 



\section{Industrial application}

\subsection{Topology optimization of a landing gear part}

In order to show a typical application for which the optimization techniques in this work are developed, a part from an early development phase is chosen and optimized. The lower part of the torque link on a main landing gear, as shown in Figure 23, was chosen because it is easily visualized and has very simple boundary conditions. The aim with this example is to show a possible scenario, not to optimize a design that will be used in service; the loading conditions and the structural requirements are thus somewhat fictitious.

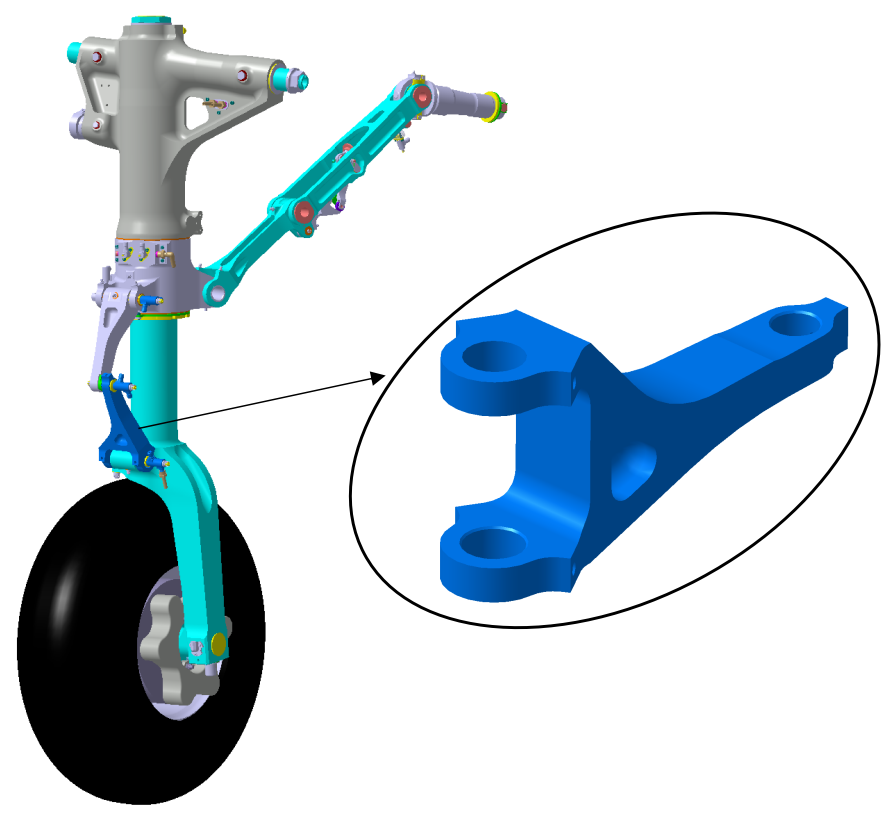

Figure 23: Landing gear with the previous design of the lower torque link.

The existing model, Figure 24(a), of the torque link was modified in Catia [62] to create a relevant design domain for the topology optimization. The outer shape was 
kept due to clearance, but the cut-outs were filled and the radii were replaced by slanted edges. The simplified Catia model of the design domain was then imported into HyperMesh where it was discretized using eight-node hexahedral and six-node pentahedral elements, in total 39313 solid elements were used and the mesh is shown in Figure 24(b). The boundary conditions are applied at the center of the holes, from which rigid body elements connect to the inner surface of the holes. At the hole where the load is applied the translation in the $y$-direction is fixed and in the two other holes all translations as well as the rotations about the $x$ and $y$-axes are fixed. It was decided that the attachment lugs would not to be part of the design domain; therefore a superelement was created for the non-design elements and used in the optimization. The initial design used in the Matlab program is seen in figure $24(\mathrm{c})$.

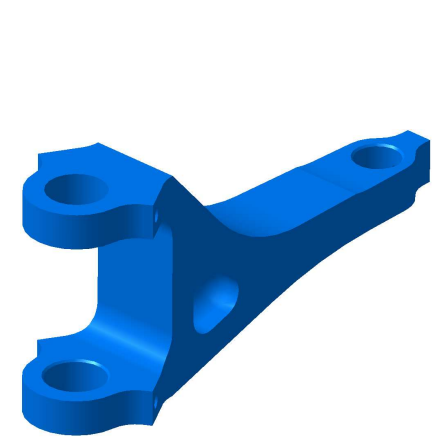

(a)

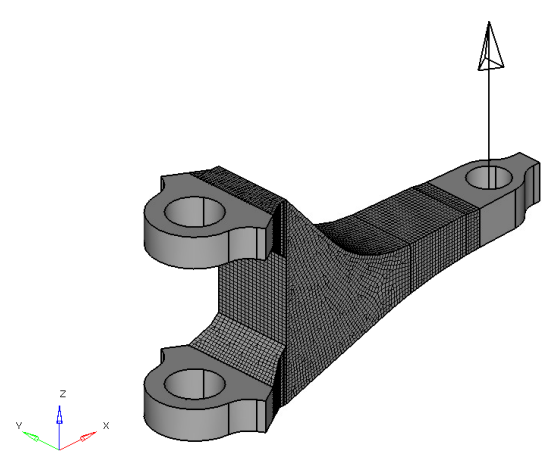

(b)

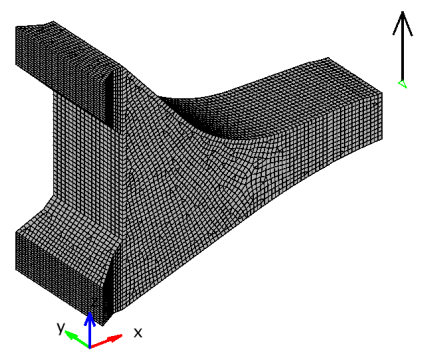

(c)

Figure 24: a: Original model in Catia, b: Design and non-design spaces defined in HyperMesh, c: Discretized design domain imported in Matlab.

The torque link transfers rotation about the axis of the strut in Figure 23, and the only loads it is supposed to take are in the $z$-direction in Figure 24(c). Here, the von Mises stress measure will be used, meaning that no distinction is needed between loads in the positive or negative $z$-direction; therefore only the maximum absolute value of the loads is used in the optimization. This load is $\hat{\boldsymbol{f}}$. 
However, due to friction and jamming a load in the $x$-direction may occur, as well as combinations between the $z$ and $x$-directions. Therefore, robust optimization with respect to an uncertain loading was performed. The load was varying in the $x z$-plane, on the ellipse defined by $\hat{\boldsymbol{f}}$ in $z$ and $0.1 \hat{\boldsymbol{f}}$ in $x$-direction.

First, a worst-case compliance optimization was performed using the SDP-formulation (24). The mass constraint limit was chosen to $15 \%$ of the mass of the full design space. The worst-case compliance design is seen in Figure 25 and a non-robust design is seen in Figure 26. The robust design is a more solid topology compared to the non-robust design, for which the topology is divided into two main parts.

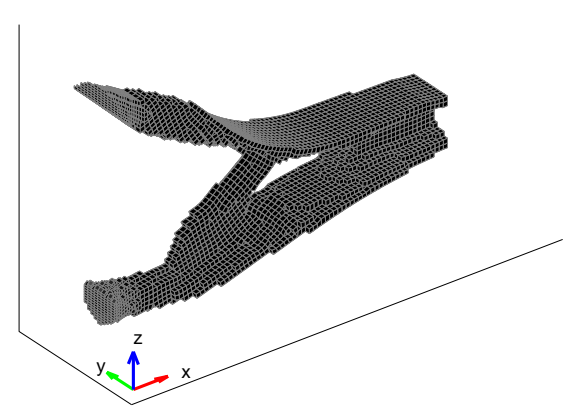

(a)

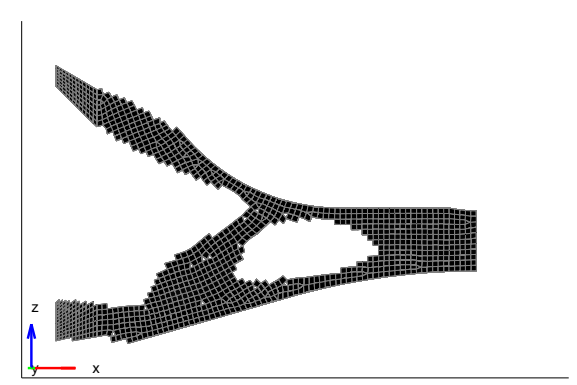

(b)

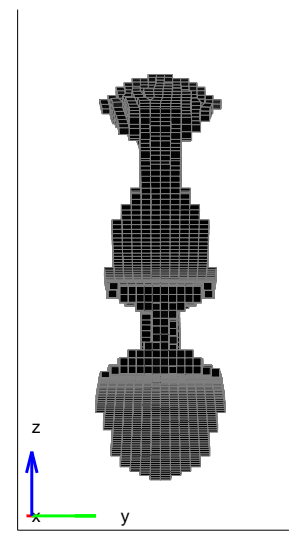

(c)

Figure 25: Robust design obtained for a worst-case compliance minimization problem. a: Iso-view, b: Sideview, c: Frontview.

Obviously, robustness is obtained at the price of a higher compliance for any load compared to a compliance minimization performed solely for that specific load; however, in this case a better local optimum was found by the SDP-formulation than the standard compliance formulation, which was solved using MMA. The compliance for a load in the $z$-direction is actually slightly lower for the robust 


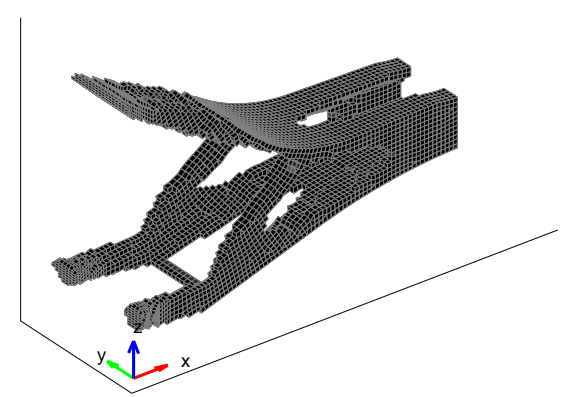

Figure 26: Design obtained for a non-robust compliance minimization problem where the load is in the $z$-direction.

design in Figure 25, it is $0.998 C$, where $C$ is the compliance for the non-robust design in Figure 26.

The problem with the compliance based design is the lack of control over the stresses and that it may be difficult to choose an appropriate mass limit. Therefore, the game theory approach is also used to find a minimum mass design where the global stress measure (14) is below the stress limit $\bar{\sigma}$, for the same load uncertainty as above. The load variable problem is now chosen to maximize the global stress measure. The optimized design is seen in Figure 27. The maximum local von Mises stress is now approximately $0.85 \bar{\sigma}$, as is shown in Figure $27(\mathrm{~d})$. As the mass in the optimized design is higher (17\% of the mass of the full design space) than that used in the compliance based optimizations, the compliance for a load in the $z$-direction is lower, $0.95 C$, for the stress constrained design.

In Figure 28, the design in Figure 27 is shown together with the attachment lugs in HyperMesh. The optimized design was imported into HyperMesh from the in-data file created in the Matlab program, where elements with a design variable value lower than 0.8 were removed. For the design criteria used in this example, some adjustments of the attachment lugs would be possible to reduce the mass.

In this example, the robust compliance and robust stress constrained formulations resulted in quite similar designs. In addition, the compliance based design is almost a subset of the stress constrained design, this gives confidence in that the stress constrained topology is a good design which should be suggested as a start for the following design stages. 


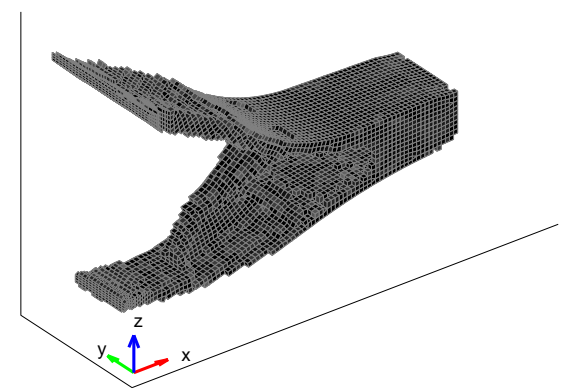

(a)

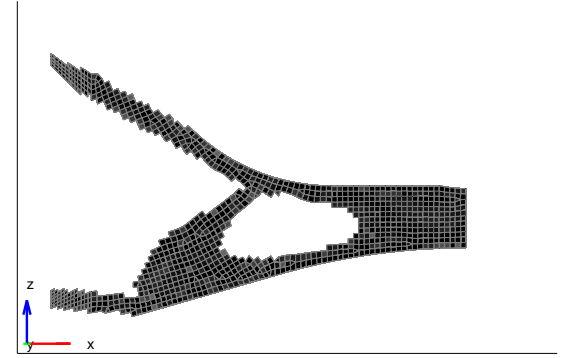

(b)

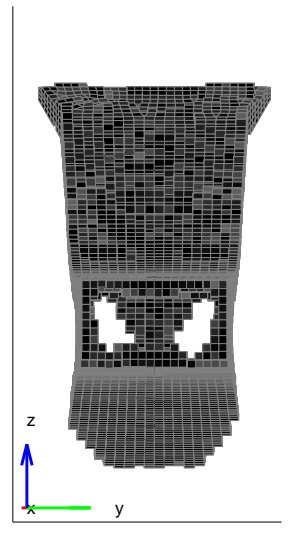

(c)

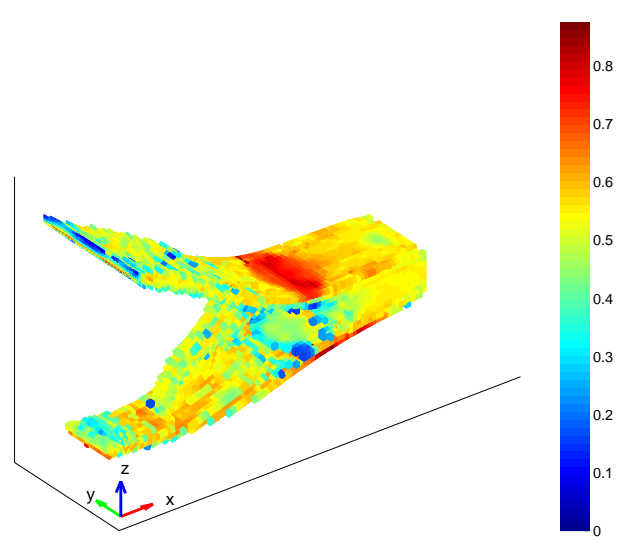

(d)

Figure 27: Robust design obtained by solving the game theory problem described above. a: Iso-view, b: Sideview, c: Frontview, d: von Mises stresses divided by $\bar{\sigma}$, for the load defined by $\boldsymbol{\theta}^{*}$.

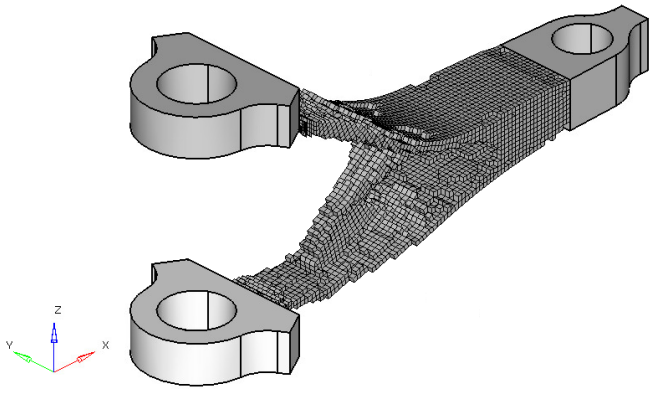

Figure 28: The design in Figure 27, exported to HyperMesh and shown together with the non-design volumes. 



\section{Summary and conclusions}

This work presents a step towards more advanced topology optimizations in conceptual design stages, with the aim of obtaining better designs and facilitating the further design work. The main objective has been to generate light-weight conceptual designs that are designed with respect to structural requirements such as stress and fatigue, and that are robust with respect to load uncertainties, so that they still satisfy their requirements for possible variations of the load.

The clustered approach that has been developed for stress constraints gives a tradeoff between computational cost and accuracy and has proven to work well. Large stress concentrations are avoided and the stresses in the optimized conceptual designs are reasonably close to the specified limit. The introduced methodology for high-cycle fatigue constraints with variable-amplitude loading, allows the topology to be optimized with respect to a specific life, in a manner similar to that used for stress constraints. The method involves a few simplifications, but the effect of these are not expected to be more severe than the effect of errors introduced in the load history and the material data, or by the difference in the stress evaluated in the topology design compared to that in the actual continuum.

The SDP-formulation provides a computationally tractable and efficient formulation for solving worst-case compliance problems. Only one factorization of the stiffness matrix is required in each iteration and the main computational cost is for solving a system of linear equations where the number of right hand sides equals the spatial dimension plus one, which means that it can be compared to solving the state equation for the same number of load cases. The game theoretic framework introduced allows the formulation of robust topology optimization for a very wide range of problems; for example, stress constrained mass minimization problems have been solved with uncertain loading which was chosen such that the global stress was maximized.

The computational cost has always been included as one important aspect when the methods have been developed, but is obviously higher than for the traditional stiffness based problem formulation. However, even though the optimization requires more time, the designs obtained in this work are more mature and the total product development time is therefore expected to be shorter, as the necessity of manual trial-and-error is reduced. 



\section{Review of included papers}

\section{Paper I}

\section{Stress constrained topology optimization}

In the first paper, static stress constraints based on von Mises stress criterion are introduced in topology optimization problems, where the mass is minimized or the stiffness is maximized. The main focus is on how to formulate the stress constraints such that the computational cost is reasonable while a good representation of the local stresses is still maintained. This is achieved by dividing the stress evaluation points into clusters, where a clustered stress measure is calculated. One stress constraint is then applied to each cluster instead of each stress evaluation point, which reduces the number of constraints significantly. Two different methods for sorting the stress evaluation points into the clusters are evaluated as well as whether the clusters should be fixed or updated during the iterations. Two examples are used to confirm the discussed differences between the developed methods.

\section{Paper II}

\section{Fatigue constrained topology optimization}

The second paper addresses topology optimization of structures subjected to repeated loading conditions, where a fatigue failure might occur even if the stresses are below the yield limit of the material. A fatigue analysis software is used to determine the highest stress that gives an allowable cumulative damage for prescribed variable-amplitude loading conditions during a specific life time. Fatigue constraints are then introduced in order to find a light-weight design that is dimensioned by this critical fatigue stress. A clustered approach is used where the highest tensile principal stress is used as the local stress measure, and the fatigue constraints are used together with the von Mises based static stress constraints developed in paper I. For comparison purposes, the difference between stress constraints based on von Mises and the highest tensile principal stress are also evaluated. 


\section{Paper III}

Worst-case topology optimization of self-weight loaded structures using semi-definite programming

An issue in topology optimization is that an optimized design tends to be very weak for any load other than those for which it was optimized. This issue is addressed by performing worst-case compliance optimization with uncertain loading, where the structure is optimized for the worst load from an uncertainty set. It is shown how such a problem can be formulated as a non-linear semi-definite programming problem which can be solved efficiently using an interior-point method, since the constraint matrix involved is of small size and since the problem becomes computationally tractable, which is not the case for previously existing methods. The loading is due to the acceleration of the self-weight of the optimized structure and of point masses applied to it. A new method for topology optimization with self-weight is also presented, as well as a method to obtain more black-and-white optimized designs.

\section{Paper IV}

\section{Large-scale robust topology optimization under load-uncertainty}

The paper is an extension of the method developed in paper III, but here the loading is due to external forces acting on the structure and both fixed and uncertain forces may be applied simultaneously. The problem is formulated as a non-linear semi-definite programming problem and the size of the (square) matrix constraint is only increased from $s$ to $s+1$, where $s$ is the spatial dimension. Thus, the constraint matrix is still small and the computational cost of introducing this uncertain loading is comparable to the cost of solving the state equation for $s+1$ load cases. An example is given where the fixed and uncertain loads act in the same point, which gives a design that is robust with respect to perturbations of the load direction.

\section{Paper V}

Game theory approach to robust topology optimization with uncertain loading

A game theory framework for solving robust topology optimization problems with uncertain loading is developed. The game is characterized by two players, the 
structure and the load, that antagonize and it is formulated so that a Nash equilibrium point is sought. The optimized structure is found using the standard topology optimization variables and the load is parameterized by the angles in a spherical coordinate system (in 3D), which are varied in order to find the worst possible load. The range of objective and constraint functions that can be used in this formulation is very broad and three different games are solved numerically. In the first formulation, the first player (the structure) wants to minimize the compliance and the second player (the load) wants to maximize compliance. The problem can thus be compared with the SDP-formulation in papers III and IV. The two other formulations involve global stress as objective or constraint function. 



\section{Bibliography}

[1] Saab AB. http://www.saabgroup.com.

[2] Altair. HyperMesh 13.0, users manual. Altair Engineering, 2014.

[3] J.P. Aubin. Mathematical methods of game and economic theory. NorthHolland, Amsterdam, 1979.

[4] M.P. Bendsøe. Optimal shape design as a material distribution problem. Structural optimization, 1(4):193-202, 1989.

[5] M.P. Bendsøe and N. Kikuchi. Generating optimal topologies in structural design using a homogenization method. Computer methods in applied mechanics and engineering, 71(2):197-224, 1988.

[6] M.P. Bendsøe and O. Sigmund. Material interpolation schemes in topology optimization. Archive of Applied Mechanics, 69(9):635-654, 1999.

[7] M.P. Bendsøe and O. Sigmund. Topology Optimization - Theory, Methods, and Applications. Springer Verlag, Berlin, second edition edition, 2003.

[8] T. Borrvall and J. Petersson. Topology optimization using regularized intermediate density control. Computer Methods in Applied Mechanics and Engineering, 190(37):4911-4928, 2001.

[9] K. Brittain, M. Silva, and D.A. Tortorelli. Minmax topology optimization. Structural and Multidisciplinary Optimization, 45(5):657-668, 2012.

[10] M. Bruggi. On an alternative approach to stress constraints relaxation in topology optimization. Structural and multidisciplinary optimization, 36(2):125-141, 2008.

[11] T.E. Bruns. A reevaluation of the simp method with filtering and an alternative formulation for solid-void topology optimization. Structural and Multidisciplinary Optimization, 30(6):428-436, 2005.

[12] T.E. Bruns and D.A. Tortorelli. Topology optimization of non-linear elastic structures and compliant mechanisms. Computer Methods in Applied Mechanics and Engineering, 190(26-27):3443-3459, 2001.

[13] G. Cheng and Z. Jiang. Study on topology optimization with stress constraints. Engineering Optimization, 20(2):129-148, 1992. 
[14] G.D. Cheng and X. Guo. $\varepsilon$-relaxed approach in structural topology optimization. Structural and Multidisciplinary Optimization, 13(4):258-266, 1997.

[15] K-T. Cheng and N. Olhoff. An investigation concerning optimal design of solid elastic plates. International Journal of Solids and Structures, 17(3):305-323, 1981.

[16] E. Cherkaev and A. Cherkaev. Minimax optimization problem of structural design. Computers \& Structures, 86(13):1426-1435, 2008.

[17] M. Collet, M. Bruggi, S. Bauduin, D. Ruffoni, and P. Duysinx. Simplified fatigue resistance in mechanical engineering using topology optimization. In 9th European Solid Mechanics Conference, 2015.

[18] R.D. Cook, D.S. Malkus, M.E. Plesha, and R.J. Witt. Concepts and applications of finite element analysis. John Wiley \& Sons, 2002.

[19] T. Dahlberg and A. Ekberg. Failure, fracture, fatigue: an introduction. Studentlitteratur, 2002.

[20] B. Desmorat and R. Desmorat. Topology optimization in damage governed low cycle fatigue. Comptes Rendus Mecanique, 336(5):448-453, 2008.

[21] A. Díaz and O. Sigmund. Checkerboard patterns in layout optimization. Structural and Multidisciplinary Optimization, 10(1):40-45, 1995.

[22] W.S. Dorn, R.E. Gomory, and H.J. Greenberg. Automatic design of optimal structures. Journal de mecanique, 3(6):25-52, 1964.

[23] P. Duysinx and M.P. Bendsøe. Topology optimization of continuum structures with local stress constraints. International Journal for Numerical Methods in Engineering, 43(8):1453-1478, 1998.

[24] P. Duysinx, M. Collet, S. Bauduin, E. Tromme, L. Noel, and M. Bruggi. Topology optimization of mechanical and aerospace components subject to fatigue stress constraints. In 11th World Congress on Strucural and Multidisciplinary Optimization, 2015.

[25] P. Duysinx and O. Sigmund. New developments in handling stress constraints in optimal material distribution. American institute of aeronautics and astronautics, 4906:1501-1509, 1998.

[26] J.K Guest, J.H. Prévost, and T. Belytschko. Achieving minimum length scale in topology optimization using nodal design variables and projection functions. International Journal for Numerical Methods in Engineering, 61(2):238-254, 2004 .

[27] X. Guo, G. Cheng, and K. Yamazaki. A new approach for the solution of singular optima in truss topology optimization with stress and local buckling constraints. Structural and Multidisciplinary Optimization, 22(5):364-373, 2001. 
[28] E. Holmberg, B. Torstenfelt, and A. Klarbring. Global and clustered approaches for stress constrained topology optimization and deactivation of design variables. In 10th World Congress on Structural and Multidisciplinary Optimization, 2013.

[29] R.A. Horn and C.R. Johnson. Matrix analysis. Cambridge university press, 1985.

[30] S.H. Jeong, D-H. Choi, and G.H. Yoon. Fatigue and static failure considerations using a topology optimization method. Applied Mathematical Modelling, 39(3):1137-1162, 2015.

[31] U. Kirsch. On singular topologies in optimum structural design. Structural and Multidisciplinary Optimization, 2(3):133-142, 1990.

[32] R.V. Kohn and G. Strang. Optimal design and relaxation of variational problems. Communications on pure and applied mathematics, 39:113-137(Part I),139-182(Part II),353-377(Part III), 1986.

[33] C. Le, J. Norato, T. Bruns, C. Ha, and D. Tortorelli. Stress-based topology optimization for continua. Structural and Multidisciplinary Optimization, 41(4):605-620, 2010.

[34] M. Mrzygłód. Two-stage optimization method with fatigue constraints for thin-walled structures. Journal of Theoretical and Applied Mechanics, 48(3):567-578, 2010.

[35] M. Mrzygłód. Multi-constrained topology optimization using constant criterion surface algorithm. Bulletin of the Polish Academy of Sciences: Technical Sciences, 60(2):229-236, 2012.

[36] M. Mrzygłód and A.P. Zieliński. Numerical implementation of multiaxial highcycle fatigue criterion to structural optimization. Journal of Theoretical and Applied Mechanics, 44(3):691-712, 2006.

[37] M. Mrzyglod and A.P. Zielinski. Parametric structural optimization with respect to the multiaxial high-cycle fatigue criterion. Structural and Multidisciplinary Optimization, 33(2):161-171, 2007.

[38] R.H. Myers, D.C. Montgomery, and C.M Anderson-Cook. Response surface methodology: process and product optimization using designed experiments, volume 705. John Wiley \& Sons, 2009.

[39] J. Nash. Non-cooperative games. Annals of mathematics, pages 286-295, 1951.

[40] Optistruct. 13.0, users manual. Altair Engineering, 2014.

[41] M.L. Overton. Large-scale optimization of eigenvalues. SIAM Journal on Optimization, 2(1):88-120, 1992. 
[42] J. París, F. Navarrina, I. Colominas, and M. Casteleiro. Block aggregation of stress constraints in topology optimization of structures. Advances in Engineering Software, 41(3):433-441, 2010.

[43] N.L. Pedersen and A.K. Nielsen. Optimization of practical trusses with constraints on eigenfrequencies, displacements, stresses, and buckling. Structural and Multidisciplinary Optimization, 25(5):436-445, 2003.

[44] R.T. Rockafeller. Convex Analysis. Princeton, 1972.

[45] G.I.N. Rozvany. Aims, scope, methods, history and unified terminology of computer-aided topology optimization in structural mechanics. Structural and Multidisciplinary Optimization, 21(2):90-108, 2001.

[46] G.I.N. Rozvany. A critical review of established methods of structural topology optimization. Structural and Multidisciplinary Optimization, 37(3):217-237, 2009 .

[47] G.I.N. Rozvany and T. Birker. On singular topologies in exact layout optimization. Structural and Multidisciplinary Optimization, 8(4):228-235, 1994.

[48] G.I.N. Rozvany, M. Zhou, and T. Birker. Generalized shape optimization without homogenization. Structural and Multidisciplinary Optimization, 4(3):250 $252,1992$.

[49] J. Schijve. Fatigue of structures and materials. Springer, 2009.

[50] A.P. Seyranian, E. Lund, and N. Olhoff. Multiple eigenvalues in structural optimization problems. Structural and Multidisciplinary Optimization, 8(4):207227, 1994.

[51] O. Sigmund. On the design of compliant mechanisms using topology optimization. Journal of Structural Mechanics, 25(4):493-524, 1997.

[52] O. Sigmund. Topology optimization: a tool for the tailoring of structures and materials. Philosophical Transactions of the Royal Society of London. Series A: Mathematical, Physical and Engineering Sciences, 358(1765):211227,2000 .

[53] O. Sigmund. Morphology-based black and white filters for topology optimization. Structural and Multidisciplinary Optimization, 33(4):401-424, 2007.

[54] O. Sigmund. On the usefulness of non-gradient approaches in topology optimization. Structural and Multidisciplinary Optimization, 43:589-596, 2011.

[55] O. Sigmund and J. Petersson. Numerical instabilities in topology optimization: a survey on procedures dealing with checkerboards, mesh-dependencies and local minima. Structural and Multidisciplinary Optimization, 16(1):68-75, 1998.

[56] G. Strang and R.V. Kohn. Optimal design in elasticity and plasticity. International journal for numerical methods in engineering, 22:183-188, 1986. 
[57] S. Suresh. Fatigue of materials. Cambridge University Press, 1998.

[58] K. Svanberg. The method of moving asymptotes - a new method for structural optimization. International journal for numerical methods in engineering, 24(2):359-373, 1987.

[59] K. Svanberg and H. Svärd. Density filters for topology optimization based on the pythagorean means. Structural and Multidisciplinary Optimization, 48(5):859-875, 2013.

[60] H. Svärd. Topology optimization of fatigue-constrained structures. 2015.

[61] G. Sved and Z. Ginos. Structural optimization under multiple loading. International Journal of Mechanical Sciences, 10(10):803-805, 1968.

[62] Dassault systems. Catia v5, 2015. http://www.3ds.com/ products-services/catia.

[63] A. Takezawa, S. Nii, M. Kitamura, and N. Kogiso. Topology optimization for worst load conditions based on the eigenvalue analysis of an aggregated linear system. Computer Methods in Applied Mechanics and Engineering, 200(25):2268-2281, 2011.

[64] The MathWorks Inc. MATLAB - User's Guide. Natick, Massachusetts, United States, 2015.

[65] C-J Thore. Fminsdp - a code for solving optimization problems with matrix inequality constraints, 2013. http://www.mathworks.com/matlabcentral/ fileexchange/43643-fminsdp.

[66] B. Torstenfelt. The trinitas project, 2012. http://www.solid.iei.liu.se/ Offered $\backslash$ textunderscoreservices/Trinitas.

[67] A. Wächter and L.T. Biegler. On the implementation of an interior-point filter line-search algorithm for large-scale nonlinear programming. Mathematical programming, 106(1):25-57, 2006.

[68] Shun Wang, Eric de Sturler, and Glaucio H Paulino. Large-scale topology optimization using preconditioned krylov subspace methods with recycling. Int. J. Numer. Meth. Engng, 69:2441-2468, 2007.

[69] R.J. Yang and C.J. Chen. Stress-based topology optimization. Structural and Multidisciplinary Optimization, 12(2):98-105, 1996.

[70] M. Zhou, Y.K. Shyy, and H.L. Thomas. Checkerboard and minimum member size control in topology optimization. Structural and Multidisciplinary Optimization, 21(2):152-158, 2001. 


\section{Part II}

\section{Included papers}





\section{Papers}

The articles associated with this thesis have been removed for copyright reasons. For more details about these see:

http://urn.kb.se/resolve?urn=urn:nbn:se:liu:diva-123008 